\title{
Holographic DC conductivity for backreacted NLED in massive gravity
}

\author{
Shihao Bi and Jun Tao \\ Center for Theoretical Physics, College of Physics, Sichuan University, \\ Chengdu, 610065, China \\ E-mail: bishihao@stu.scu.edu.cn, taojun@scu.edu.cn
}

\begin{abstract}
In this work a holographic model with the charge current dual to a general nonlinear electrodynamics (NLED) is discussed in the framework of massive gravity. Massive graviton can break the diffeomorphism invariance in the bulk and generates momentum dissipation in the dual boundary theory. The expression of DC conductivities in a finite magnetic field are obtained, with the backreaction of NLED field on the background geometry. General transport properties in various limits are presented, and then we turn to the three of specific NLED models: the conventional Maxwell electrodynamics, the Maxwell-Chern-Simons electrodynamics, and the Born-Infeld electrodynamics, to study the parameter-dependence of in-plane resistivities. Two mechanisms leading to the Mottinsulating behaviors and negative magneto-resistivities are revealed at zero temperature, and the role played by the massive gravity coupling parameters are discussed.
\end{abstract}

KEYwORDS: AdS-CFT Correspondence, Black Holes

ArXiv EPrint: 2101.00912 


\section{Contents}

1 Introduction 1

2 Holographic setup 3

3 DC conductivity 5

3.1 Derivation of DC conductivity 5

3.2 Various limits 7

$\begin{array}{lll}3.2 .1 & \text { Massless and massive limits } & 7\end{array}$

$\begin{array}{lll}3.2 .2 & \text { Zero field and charge density limits } & 7\end{array}$

3.2.3 High temperature limit 8

4 Various NLED models $\quad 9$

4.1 Maxwell electrodynamics 9

$\begin{array}{ll}4.2 & \text { Maxwell-Chern-Simons electrodynamics } \\ & 12\end{array}$

$\begin{array}{lll}4.3 & \text { Born-Infeld electrodynamics } & 16\end{array}$

$\begin{array}{lll}5 & \text { Conclusion } & 19\end{array}$

$\begin{array}{ll}\text { A Consistency of metric ansatz } & 21\end{array}$

\section{Introduction}

The discovery of gauge/gravity duality makes it possible to deal with the strongly-coupled gauge theories on the boundary from the classical gravitational theories in the higher dimensional bulk [1-6]. And the well-known prediction on the ratio of the shear viscosity to the entropy density for $\mathcal{N}=4$ super Yang-Mills (SYM) theory was found to be close to the experimental results of real quark-gluon plasma (QGP) [7-10], making it more convincing to physical community. In recent years the idea of gauge/gravity duality has been applied to hydrodynamics [11-14], quantum chromodynamics (QCD) [15-24], nuclear physics [25, 26], and strongly-coupled condensed matter systems [27-37], etc., and new insights are brought into these physical branches.

The establishment of quantum many-body theory [38, 39] in a fairly low energy scale compared with high energy physics is another magnificent and fascinating story apart from that of the standard model, which have profoundly extended and deepen our understanding of realistic matters. Many characterization methods, based on the linear response theory or quantum transport theory, have been developed in the scattering or transport experiments, to reveal the electronic or lattice structures, surface topography, defects and disorder, and study the transport properties of the material samples. In the framework of the energy band 
theory the materials are roughly divided into three categories: metals, semiconductors, and insulators, which differs in their electrical conductivities. And it is known to all of us the classical electron motion in the presence of the magnetic field has been studied since 1879, which is the famous classical Hall effect [40]. Its quantum version, known as the quantum Hall effect, was first observed in 1980 by von Klitzing [41], has aroused a wide research enthusiasm in the last two decades and witnessed impressive theoretical and experimental breakthroughs $[42,43]$. The new quantum phases of matter, termed topological insulators, exhibit another kind of bulk-boundary correspondence between the gapped insulated bulk and gapless metallic edge states on the boundary. Its exotic transport properties have also attracted lots of research interests, among which the conductivity behavior in the presence of magnetic field is of vital importance, for it provides a promising way on controlling the electrical properties with the help of external fields. For normal metals the resistivity always increases with the magnetic field strength [44], showing the positive magneto-resistivity. However, the experimental measurements [45-49] in topological materials, such as Dirac or Weyl semimetals, etc., demonstrated the presence of negative magneto-resistivity or crossover for positive to negative, which are mainly attributed to chiral anomaly [50-54]. In addition, the negative magneto-resistivity behaviors are also found in holographic chiral anomalous systems [55-58].

Mott insulators, which are believed to be parent materials of cuprate high $T_{c}$ superconductors [59], is another example beyond the framework of energy band theory due to the strong Coulomb repulsive interaction. The strong electron-electron interaction would prevent the available charge carriers to efficiently transport charges as if suffers the electronic traffic jam. The holographic construction of Mott insulators has been a heated topic and much progress has been made [32, 60-67]. In ref. [68], the holographic model coupled with a particular NLED named iDBI was proposed to study the strong electron-electron interaction by introducing the self-interaction of NLED field, and the Mott-insulating behaviors appear for large enough self-interaction strength.

In this paper, we construct a holographic model coupled with a general NLED field to investigate the magneto-transport of the strongly-interacting system on the boundary from the perspective of gauge/gravity duality. The backreaction of NLED field on the bulk geometry is taken into consideration as in ref. [69]. The massive gravity, with massive potentials associated with the graviton mass, is adopted to break the diffeomorphism invariance in the bulk producing momentum relaxation in the dual boundary theory [70]. This model can serve as an effective description of strange metal phase and exhibiting the negative magneto-resistivities and Mott-insulating behaviors in certain circumstance.

This paper is organized as follows. In section 2 we establish our holographic model of massive gravity with NLED field. Then the DC conductivities with non-zero magnetic field are derived as the function of $\rho, h, T$, and related massive gravity coupling parameters in section 3 , with various limit situation being discussed. Then in section 4 we present detailed investigation on the in-plane resistivities in the framework of conventional Maxwell electrodynamics, the CP-violating Maxwell-Chern-Simons electrodynamics, and Born-Infeld electrodynamics. Finally, we make our conclusion in section 5 . We will use the unit $\hbar=G=k_{B}=l=1$. 


\section{Holographic setup}

The 4-dimensional massive gravity [70] with a negative cosmological constant $\Lambda$ coupled to a nonlinear electromagnetic field $A_{\mu}$ we are considering is given by

$$
S=\frac{1}{16 \pi} \int \mathrm{d}^{4} x \sqrt{-g}\left[R-2 \Lambda+m^{2} \sum_{j=1}^{4} c_{j} \mathcal{U}_{j}(g, f)+\mathcal{L}(s, p)\right],
$$

where $\Lambda=-3 / l^{2}, m$ is the mass parameter, $f$ is a fixed symmetric tensor called the reference metric, $c_{j}$ are coupling constants, ${ }^{1}$ and $\mathcal{U}_{j}(g, f)$ denotes the symmetric polynomials of the eigenvalue of the $4 \times 4$ matrix $\mathcal{K}_{\nu}^{\mu}=\sqrt{g^{\mu \lambda} f_{\lambda \nu}}$ given as

$$
\begin{aligned}
& \mathcal{U}_{1}=[\mathcal{K}], \\
& \mathcal{U}_{2}=[\mathcal{K}]^{2}-\left[\mathcal{K}^{2}\right], \\
& \mathcal{U}_{3}=[\mathcal{K}]^{3}-3[\mathcal{K}]\left[\mathcal{K}^{2}\right]+2\left[\mathcal{K}^{3}\right], \\
& \mathcal{U}_{4}=[\mathcal{K}]^{4}-6[\mathcal{K}]^{2}\left[\mathcal{K}^{2}\right]+8[\mathcal{K}]\left[\mathcal{K}^{3}\right]+3\left[\mathcal{K}^{2}\right]^{2}-6\left[\mathcal{K}^{4}\right] .
\end{aligned}
$$

The square root in $\mathcal{K}$ means $(\sqrt{\mathcal{K}})_{\lambda}^{\mu}(\sqrt{\mathcal{K}})_{\nu}^{\lambda}=\mathcal{K}_{\nu}^{\mu}$ and $[\mathcal{K}] \equiv \mathcal{K}_{\mu}^{\mu}$. The NLED Lagrangian $\mathcal{L}(s, p)$ in eq. (2.1) is constructed as the function of two independent nontrivial scalar using the field strength tensor $F_{\mu \nu}=\partial_{\mu} A_{\nu}-\partial_{\nu} A_{\mu}$

$$
\begin{aligned}
& s=-\frac{1}{4} F^{\mu \nu} F_{\mu \nu}, \\
& p=-\frac{1}{8} \varepsilon^{\mu \nu \rho \sigma} F_{\mu \nu} F_{\rho \sigma} .
\end{aligned}
$$

Here $\epsilon^{a b c d} \equiv-[a b c d] / \sqrt{-g}$ is a totally antisymmetric Lorentz tensor, and $[a b c d]$ is the permutation symbol. In the weak field limit we assume the NLED reduces to the Maxwell-Chern-Simons Lagrangian $\mathcal{L}(s, p) \approx s+\theta p$ with $\theta$ defined as $\mathcal{L}^{(0,1)}(0,0)$ for later convenience.

Varying the action with respect to $g^{\mu \nu}$ and $A^{\mu}$ we obtain the equations of motion

$$
\begin{aligned}
R_{\mu \nu}-\frac{1}{2} R g_{\mu \nu}+\Lambda g_{\mu \nu} & =\frac{1}{2} T_{\mu \nu}, \\
\nabla_{\mu} G^{\mu \nu} & =0,
\end{aligned}
$$

where the energy-momentum tensor is

$$
T_{\mu \nu}=g_{\mu \nu}\left(\mathcal{L}(s, p)-p \frac{\partial \mathcal{L}(s, p)}{\partial p}\right)+\frac{\partial \mathcal{L}(s, p)}{\partial s} F_{\mu}^{\lambda} F_{\nu \lambda}+m^{2} \chi_{\mu \nu},
$$

with

$$
\begin{aligned}
\chi_{\mu \nu}= & c_{1}\left(\mathcal{U}_{1} g_{\mu \nu}-\mathcal{K}_{\mu \nu}\right)+c_{2}\left(\mathcal{U}_{2} g_{\mu \nu}-2 \mathcal{U}_{1} \mathcal{K}_{\mu \nu}+2 \mathcal{K}_{\mu \nu}^{2}\right) \\
& +c_{3}\left(\mathcal{U}_{3} g_{\mu \nu}-3 \mathcal{U}_{2} \mathcal{K}_{\mu \nu}+6 \mathcal{U}_{1} \mathcal{K}_{\mu \nu}^{2}-6 \mathcal{K}_{\mu \nu}^{3}\right) \\
& +c_{4}\left(\mathcal{U}_{4} g_{\mu \nu}-4 \mathcal{U}_{3} \mathcal{K}_{\mu \nu}+12 \mathcal{U}_{2} \mathcal{K}_{\mu \nu}^{2}-24 \mathcal{U}_{1} \mathcal{K}_{\mu \nu}^{3}+24 \mathcal{K}_{\mu \nu}^{4}\right)
\end{aligned}
$$

\footnotetext{
${ }^{1}$ To make the massive gravity theory self-consistent, all the $c_{j}$ will be set to be negative.
} 
And we introduce

$$
G^{\mu \nu}=-\frac{\partial \mathcal{L}(s, p)}{\partial F_{\mu \nu}}=\frac{\partial \mathcal{L}(s, p)}{\partial s} F^{\mu \nu}+\frac{1}{2} \frac{\partial \mathcal{L}(s, p)}{\partial p} \varepsilon^{\mu \nu \rho \sigma} F_{\rho \sigma} .
$$

We are looking for a black brane solution with asymptotic AdS spacetime by taking the following ansatz $[70,71]$ for the metric and the NLED field, and the reference metric:

$$
\begin{aligned}
\mathrm{d} s^{2} & =-f(r) \mathrm{d} t^{2}+\frac{\mathrm{d} r^{2}}{f(r)}+r^{2}\left(\mathrm{~d} x^{2}+\mathrm{d} y^{2}\right), \\
A & =A_{t}(r) \mathrm{d} t+\frac{h}{2}(x \mathrm{~d} y-y \mathrm{~d} x) \\
f_{\mu \nu} & =\operatorname{diag}\left(0,0, \alpha^{2}, \alpha^{2}\right) .
\end{aligned}
$$

where $h$ is the magnetic field strength. The validity of eq. (2.8a) will be demonstrated in the appendix. From eqs. (2.8a) and (2.8b) we find the nontrivial scalars eq. (2.3) are

$$
\begin{aligned}
& s=\frac{1}{2}\left(A_{t}^{\prime}(r)^{2}-\frac{h^{2}}{r^{4}}\right), \\
& p=-\frac{h A_{t}^{\prime}(r)}{r^{2}} .
\end{aligned}
$$

Then the equations of motion are obtained as

$$
\begin{aligned}
r f^{\prime}(r)+f(r)-3 r^{2} & =c_{1} \alpha m^{2} r+c_{2} \alpha^{2} m^{2}+\frac{r^{2}}{2}\left(A_{t}^{\prime}(r) G^{r t}+\mathcal{L}(s, p)\right) \\
r f^{\prime \prime}(r)+2 f^{\prime}(r)-6 r & =c_{1} \alpha m^{2}+r\left(\mathcal{L}(s, p)+h G^{x y}\right) \\
{\left[r^{2} G^{r t}\right]^{\prime} } & =0
\end{aligned}
$$

where the non-vanishing components $G^{\mu \nu}$ are

$$
\begin{aligned}
G^{r t} & =\frac{\partial \mathcal{L}}{\partial p} \frac{h}{r^{2}}-\frac{\partial \mathcal{L}}{\partial s} A_{t}^{\prime}(r), \\
G^{x y} & =\frac{\partial \mathcal{L}}{\partial s} \frac{h}{r^{4}}+\frac{\partial \mathcal{L}}{\partial p} \frac{A_{t}^{\prime}(r)}{r^{2}} .
\end{aligned}
$$

eq. (2.10c) leads to $G^{t r}=\rho / r^{2}$ with $\rho$ being a constant. The event horizon $r_{h}$ is the root of $f(r)$, i.e., $f\left(r_{h}\right)=0$, and the Hawking temperature of the black brane is given by

$$
T=\frac{f^{\prime}\left(r_{h}\right)}{4 \pi} .
$$

Then at $r=r_{h}$ eq. (2.10a) reduces to

$$
4 \pi T r_{h}-3 r_{h}^{2}=c_{1} \alpha m^{2} r_{h}+c_{2} \alpha^{2} m^{2}+\frac{r_{h}^{2}}{2}\left(A_{t}^{\prime}\left(r_{h}\right) G_{h}^{r t}+\mathcal{L}\left(s_{h}, p_{h}\right)\right)
$$

where

$$
\begin{aligned}
s_{h} & =\frac{1}{2}\left(A_{t}^{\prime}\left(r_{h}\right)^{2}-\frac{h^{2}}{r_{h}^{4}}\right) \\
p_{h} & =-\frac{h A_{t}^{\prime}\left(r_{h}\right)}{r_{h}^{2}} \\
G_{h}^{r t} & =\mathcal{L}^{(0,1)}\left(s_{h}, p_{h}\right) \frac{h}{r_{h}^{2}}-\mathcal{L}^{(1,0)}\left(s_{h}, p_{h}\right) A_{t}^{\prime}\left(r_{h}\right)
\end{aligned}
$$




\section{DC conductivity}

From the perspective of gauge/gravity duality, the black brane solution eqs. (2.8a) and $(2.8 \mathrm{~b})$ in the bulk can describe an equilibrium state at finite temperature $T$ given by eq. (2.13). And the conserved current $\mathcal{J}^{\mu}$ in the boundary theory is connected with the conjugate momentum of the NLED field in the bulk, which allows us to calculate the DC conductivity in the framework of linear response theory $[72,73]$.

\subsection{Derivation of DC conductivity}

The following perturbations on the metric and the NLED field are applied to derive the DC conductivity:

$$
\begin{aligned}
\delta g_{t i} & =r^{2} h_{t i}(r), \\
\delta g_{r i} & =r^{2} h_{r i}(r), \\
\delta A_{i} & =-E_{i} t+a_{i}(r),
\end{aligned}
$$

where $i=x, y$. We first consider the $t$ component. The absence of $A_{t}(r)$ in the eq. $(2.9 \mathrm{~b})$ leads to the radially independent conjugate momentum $\partial_{r} \Pi^{i}=0$, with

$$
\Pi^{t}=\frac{\partial \mathcal{L}(s, p)}{\partial\left(A_{t}^{\prime}(r)\right)} .
$$

Then the expectation value of $\mathcal{J}^{t}$ in the dual boundary field theory is given by

$$
\left\langle\mathcal{J}^{t}\right\rangle=\Pi^{t}
$$

In the linear level we have $\left\langle\mathcal{J}^{t}\right\rangle=\rho$, which indicate that $\rho$ can be interpreted as the charge density in the dual field theory. At the event horizon $r=r_{h}$ the charge density $\rho$ is given by

$$
\rho=\mathcal{L}^{(1,0)}\left(s_{h}, p_{h}\right) r_{h}^{2} A_{t}^{\prime}\left(r_{h}\right)-\mathcal{L}^{(0,1)}\left(s_{h}, p_{h}\right) h .
$$

Then we consider the planar components. The NLED is explicitly independent of $a_{i}(r)$, making the conjugate momentum of the field $a_{i}(r)$

$$
\Pi^{i}=\frac{\partial \mathcal{L}(s, p)}{\partial\left(a_{i}^{\prime}(r)\right)}=\frac{\partial \mathcal{L}(s, p)}{\partial\left(\partial_{r} A_{i}\right)}=\sqrt{-g} G^{i r},
$$

being radially independent as well. And the charge currents in the dual field theory are given by $\left\langle\mathcal{J}^{i}\right\rangle=\Pi^{i}$, and can be expressed with the perturbed metric and field components $h_{t i}, h_{r i}, a_{i}^{\prime}$ and $E_{i}$ :

$$
\begin{aligned}
& \left\langle\mathcal{J}^{x}\right\rangle=-\mathcal{L}^{(1,0)}(s, p)\left[f(r) a_{x}^{\prime}(r)+h f(r) h_{r y}(r)+r^{2} A_{t}^{\prime}(r) h_{t x}(r)\right]-\mathcal{L}^{(0,1)}(s, p) E_{y} \\
& \left\langle\mathcal{J}^{y}\right\rangle=-\mathcal{L}^{(1,0)}(s, p)\left[f(r) a_{y}^{\prime}(r)-h f(r) h_{r x}(r)+r^{2} A_{t}^{\prime}(r) h_{t y}(r)\right]+\mathcal{L}^{(0,1)}(s, p) E_{x}
\end{aligned}
$$


The perturbed metric components $h_{t i}$ and $h_{r i}$ are coupled to $E_{i}$ in gravitational field equation and can be reduced. Thus we take the first order of the perturbed Einstein's equations of $t x$ and ty components and we get:

$$
\begin{aligned}
& \left(\frac{h^{2}}{r^{2}}-\frac{\alpha m^{2} c_{1} r}{\mathcal{L}^{(1,0)}(s, p)}\right) h_{t x}(r)-h A_{t}^{\prime}(r) f(r) h_{r y}(r)=A_{t}^{\prime}(r) f(r) a_{x}^{\prime}(r)-\frac{E_{y} h}{r^{2}}, \\
& \left(\frac{h^{2}}{r^{2}}-\frac{\alpha m^{2} c_{1} r}{\mathcal{L}^{(1,0)}(s, p)}\right) h_{t y}(r)+h A_{t}^{\prime}(r) f(r) h_{r x}(r)=A_{t}^{\prime}(r) f(r) a_{y}^{\prime}(r)+\frac{E_{x} h}{r^{2}} .
\end{aligned}
$$

Next by using the regularity constraints of the metric and field near the event horizon [73]:

$$
\begin{aligned}
f(r) & =4 \pi T\left(r-r_{h}\right)+\cdots, \\
A_{t}(r) & =A_{t}^{\prime}\left(r_{h}\right)\left(r-r_{h}\right)+\cdots, \\
a_{i}(r) & =-\frac{E_{i}}{4 \pi T} \ln \left(r-r_{h}\right)+\cdots, \\
h_{r i}(r) & =\frac{h_{t i}(r)}{f(r)}+\cdots,
\end{aligned}
$$

the eqs. (3.7a) and (3.7b) at the event horizon $r=r_{h}$ are

$$
\begin{aligned}
& h A_{t}^{\prime}\left(r_{h}\right) h_{t y}\left(r_{h}\right)-\left(\frac{h^{2}}{r_{h}^{2}}-\frac{\alpha m^{2} c_{1} r_{h}}{\mathcal{L}^{(1,0)}\left(s_{h}, p_{h}\right)}\right) h_{t x}\left(r_{h}\right)=A_{t}^{\prime}\left(r_{h}\right) E_{x}+\frac{h}{r_{h}^{2}} E_{y}, \\
& \left(\frac{h^{2}}{r_{h}^{2}}-\frac{\alpha m^{2} c_{1} r_{h}}{\mathcal{L}^{(1,0)}\left(s_{h}, p_{h}\right)}\right) h_{t y}\left(r_{h}\right)+h A_{t}^{\prime}\left(r_{h}\right) h_{t x}\left(r_{h}\right)=\frac{h}{r_{h}^{2}} E_{x}-A_{t}^{\prime}\left(r_{h}\right) E_{y} .
\end{aligned}
$$

Solving eqs. (3.9a) and (3.9b) for $h_{t i}\left(r_{h}\right)$ in terms of $E_{i}$ and inserting into eqs. (3.6a) and (3.6b), one can evaluate the current $\left\langle\mathcal{J}^{i}\right\rangle$ to the electric fields $E_{i}$ at the event horizon $r=r_{h}$ via $\left\langle\mathcal{J}^{i}\right\rangle=\sigma_{i j} E_{j}$, where the DC conductivities are given by

$$
\begin{gathered}
\sigma_{x x}=\sigma_{y y}=\frac{\alpha m^{2} c_{1} r_{h}\left(\frac{\alpha m^{2} c_{1} r_{h}}{\mathcal{L}^{(1,0)}\left(s_{h}, p_{h}\right)}-\frac{h^{2}}{r_{h}^{2}}+r_{h}^{2} A_{t}^{\prime 2}\left(r_{h}\right)\right)}{\left(\frac{\alpha m^{2} c_{1} r_{h}}{\mathcal{L}^{(1,0)}\left(s_{h}, p_{h}\right)}-\frac{h^{2}}{r_{h}^{2}}\right)^{2}+h^{2} A_{t}^{\prime 2}\left(r_{h}\right)}, \\
\sigma_{x y}=-\sigma_{y x}=\frac{\mathcal{L}^{(1,0)}\left(s_{h}, p_{h}\right) r_{h}^{2} A_{t}^{\prime}\left(r_{h}\right)}{h}\left(1-\frac{\left(\frac{\alpha m^{2} c_{1} r_{h}}{\mathcal{L}^{(1,0)}\left(s_{h}, p_{h}\right)}\right)^{2}}{\left(\frac{\alpha m^{2} c_{1} r_{h}}{\mathcal{L}^{(1,0)}\left(s_{h}, p_{h}\right)}-\frac{h^{2}}{r_{h}^{2}}\right)^{2}+h^{2} A_{t}^{\prime 2}\left(r_{h}\right)}\right)-\mathcal{L}^{(0,1)}\left(s_{h}, p_{h}\right) .
\end{gathered}
$$

The resistivity matrix is the inverse of the conductivity matrix:

$$
R_{x x}=R_{y y}=\frac{\sigma_{x x}}{\sigma_{x x}^{2}+\sigma_{x y}^{2}} \quad \text { and } \quad R_{x y}=-R_{y x}=\frac{\sigma_{x y}}{\sigma_{x x}^{2}+\sigma_{x y}^{2}} .
$$

The event horizon radius $r_{h}$ is the solution of eq. (2.13) and will be affected by the temperature $T$, the charge density $\rho$, the magnetic field $h$ and the parameters $c_{1,2}, m$ and $\alpha$. For a given Lagrangian $\mathcal{L}(s, p)$ one first solve $A_{t}^{\prime}\left(r_{h}\right)$ from eq. (3.4), and then plug it into eq. (2.13) to solve $r_{h}$, which will be brought into eqs. (3.10a) and (3.10b) to obtain the DC conductivity as a complicated function of the parameters mentioned above. 


\subsection{Various limits}

The concrete examples of NLED will be discussed in section 4 and the properties of DC resistivity are discussed in detail. Before focus on the specific NLED model we first consider some general properties of the DC conductivity in various limits.

\subsubsection{Massless and massive limits}

The massless limit corresponds to $m \rightarrow 0$ and the system will restore the Lorentz invariance. It has been shown that in a Lorentz invariant theory that the DC conductivities in the presence of a magnetic field were $\sigma_{x x}=0$ and $\sigma_{x y}=\rho / h$ [74]. For comparison, in the massless limit the DC conductivities in eqs. (3.10a) and (3.10b) become

$$
\sigma_{x x}=\frac{\alpha m^{2}\left|c_{1}\right| r_{h}^{3}}{h^{2}}+\mathcal{O}\left(m^{4}\right) \quad \text { and } \quad \sigma_{x y}=\frac{\rho}{h}+\mathcal{O}\left(m^{4}\right)
$$

And in the massive limit $m \rightarrow+\infty$, the DC conductivities have the asymptotic behaviors

$$
\sigma_{x x}=\mathcal{L}^{(1,0)}\left(s_{h}, p_{h}\right)+\mathcal{O}\left(m^{-2}\right) \quad \text { and } \quad \sigma_{x y}=-\mathcal{L}^{(0,1)}\left(s_{h}, p_{h}\right)+\mathcal{O}\left(m^{-2}\right)
$$

which agree with the calculation by treating the NLED field as a probe one [75], for the geometry is dominated by the massive terms.

\subsubsection{Zero field and charge density limits}

In the zero field limit $h=0$ case, the DC conductivities become

$$
\sigma_{x x}=\mathcal{L}^{(1,0)}\left(\frac{A_{t}^{\prime 2}\left(r_{h}\right)}{2}, 0\right)-\frac{\rho^{2}}{\alpha m^{2} c_{1} r_{h}^{3}} \quad \text { and } \quad \sigma_{x y}=-\mathcal{L}^{(0,1)}\left(\frac{A_{t}^{\prime 2}\left(r_{h}\right)}{2}, 0\right)
$$

where $A_{t}^{\prime}\left(r_{h}\right)$ is obtained by solving

$$
\rho=\mathcal{L}^{(1,0)}\left(\frac{A_{t}^{\prime 2}\left(r_{h}\right)}{2}, 0\right) r_{h}^{2} A_{t}^{\prime}\left(r_{h}\right)
$$

At zero charge density $\rho=0$, the DC conductivities become

$$
\sigma_{x x}^{-1}=\frac{1}{\mathcal{L}^{(1,0)}\left(-\frac{h^{2}}{2 r_{h}^{4}}, 0\right)}-\frac{h^{2}}{\alpha m^{2} c_{1} r_{h}^{3}} \quad \text { and } \quad \sigma_{x y}=-\mathcal{L}^{(0,1)}\left(-\frac{h^{2}}{2 r_{h}^{4}}, 0\right) .
$$

We find that the DC conductivities are in general non-zero and can be interpreted as incoherent contributions [76], known as the charge conjugation symmetric contribution $\sigma_{\text {ccs }}$. There is another contribution from explicit charge density relaxed by some momentum dissipation, $\sigma_{\text {diss }}$, depending on the charge density $\rho$. The results show that, for a general NLED model, the DC conductivities usually depend on $\sigma_{\text {diss }}$ and $\sigma_{\text {ccs }}$ in a nontrivial way. 

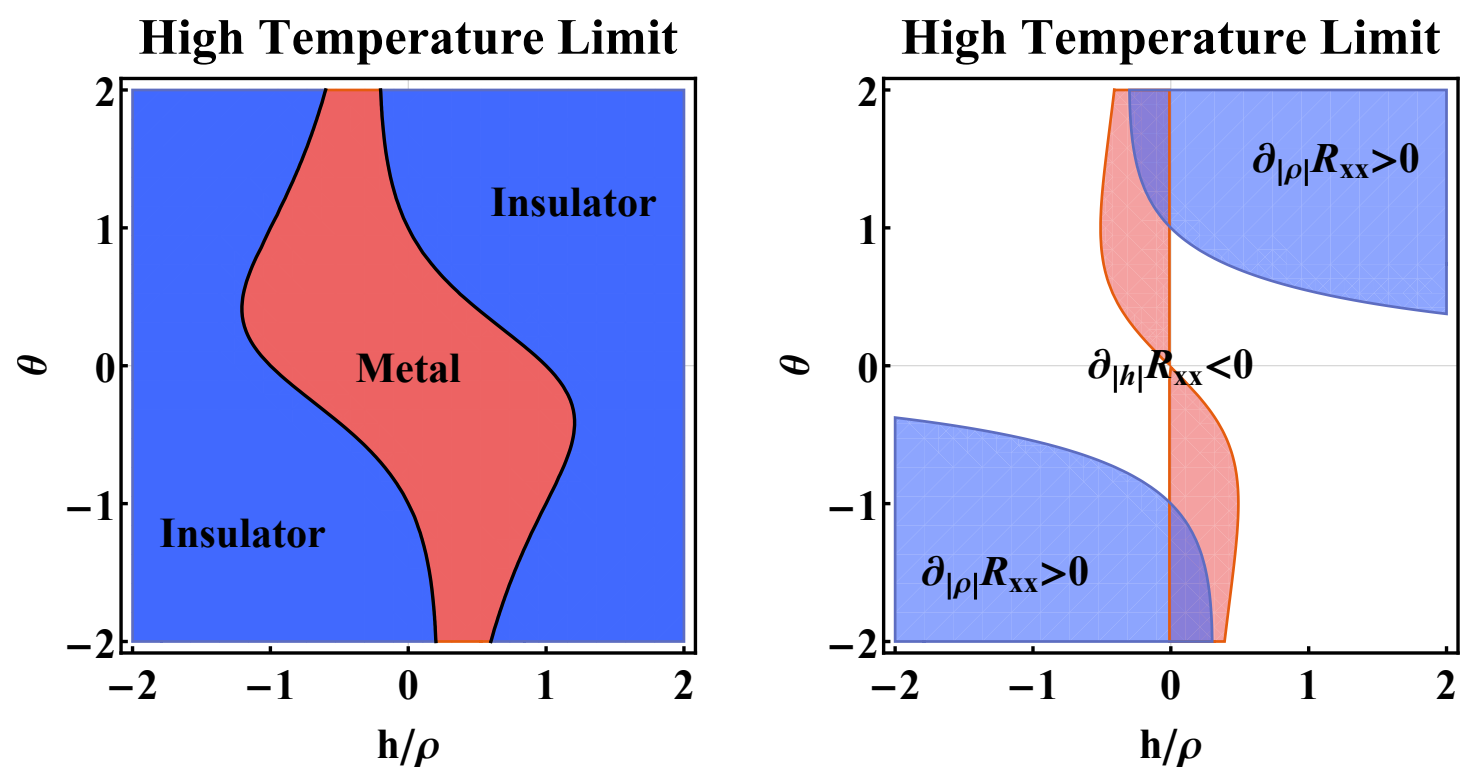

Figure 1. Left panel: the metal-insulator phase diagram in the high temperature limit. The red region has positive temperature derivative of $R_{x x}$ and thus describes a metal, while the blue region has negative temperature derivative, and hence an insulator. The black solid lines are the phase boundaries. Right panel: the Mott-insulating region (blue) and the negative magneto-resistivity (red) in the high temperature limit.

\subsubsection{High temperature limit}

Finally, we consider the high temperature limit $T \gg \max \left\{\sqrt{h}, \sqrt{\rho},\left|c_{1}\right| \alpha m^{2}, \sqrt{\left|c_{2}\right|} \alpha m\right\}$. In this limit, eq. (2.13) gives $T \approx \frac{3}{4 \pi} r_{h}$. The longitudinal resistivity then reduces to

$$
R_{x x}=\frac{1}{1+\theta^{2}}\left\{1+\frac{27}{64 \pi^{3} \alpha\left|c_{1}\right| T^{3}}\left[h^{2}\left(1+\theta^{2}\right)+2 h \theta \rho-\frac{1-\theta^{2}}{1+\theta^{2}} \rho^{2}\right]\right\}+\mathcal{O}\left(T^{-6}\right) .
$$

The nonlinear effect will be suppressed by the temperature and we only keep the leading order. Usually the DC conductivities of metal and insulator have different temperature dependence. For the metallic materials the phonon scattering enlarges the resistivity, while the thermal excitation of carriers in insulating materials can promote the conductivity. And the metal-insulator transition can occur when the coefficient of $1 / T^{2}$ changes the sign, which is shown in figure 1 in the $h / \rho-\theta$ parameter space. The $\theta$ term can break the $(\rho, h) \rightarrow(\rho,-h)$ or $(\rho, h) \rightarrow(-\rho, h)$ symmetries for $\sigma_{i j}$ or $R_{i j}$, but $\sigma_{i j}$ or $R_{i j}$ are invariant under $(\rho, h) \rightarrow(-\rho,-h)$. And the phases are central symmetric in the parameter plane.

The Mott-insulating and magneto-resistance behaviors are also presented in figure 1. The Mott-insulating behavior region is where $\partial_{|\rho|} R_{x x}>0$ in the parameter plane. The strong electron-electron interaction prevents the charge carriers from transporting. From eq. (3.17) we see that as long as $\theta^{2}>1$ the Mott-insulating behavior emerges in the absence of the magnetic field. The magneto-resistance is defined as

$$
M R_{x x}=\frac{R_{x x}(h)-R_{x x}(0)}{R_{x x}(0)},
$$


and we see that in the figure 1 the negative magneto-resistivity can only occur with non-zero $\theta$. For $\theta=0$, eq. (3.17) reduces to

$$
R_{x x}=1+\frac{27\left(h^{2}-\rho^{2}\right)}{64 \pi^{3} \alpha\left|c_{1}\right| T^{3}}+\mathcal{O}\left(T^{-6}\right)
$$

which gives metallic behavior for $|h / \rho|<1$ and insulating behavior for $|h / \rho|>1$. And one always has $\partial_{|\rho|} R_{x x}<0$ and $\partial_{|h|} R_{x x}>0$, indicating the absence of Mott-insulating behavior and negative magneto-resistivity.

\section{Various NLED models}

In this section, we will use eqs. (2.13), (3.4) and (3.10a) to study the dependence of the in-plane resistivity $R_{x x}$ on the temperature $T$, the charge density $\rho$ and the magnetic field $h$ in some specific NLED models. For convenience we rescale the $c_{i}$ 's as $c_{1} \sim \alpha m^{2} c_{1}$ and $c_{2} \sim \alpha^{2} m^{2} c_{2}$. The conventional Maxwell electrodynamics is firstly presented, with a detail discussion on the $R_{x x}$ and $R_{x y}$ 's dependence on involved massive gravity coupling parameters $c_{1}$ and $c_{2}$, the charge density $\rho$, the magnetic field $h$ and the temperature $T$. Then the Chern-Simons $\theta$ term as an extension is introduced to investigate the CPviolating effect. Finally, we discuss the Born-Infeld electrodynamics and the influence of non-linear effect on the DC resistivity. The high temperature behaviors have been discussed in section 3.2.3, so we will mainly focus on the behavior of $R_{x x}$ around $T=0$ in this section.

\subsection{Maxwell electrodynamics}

We first consider the Maxwell electrodynamics, in which $\mathcal{L}(s, p)=s$. From eq. (3.4) we can solve out that

$$
A_{t}^{\prime}\left(r_{h}\right)=\frac{\rho}{r_{h}^{2}}
$$

and bring it into eq. (2.13) we get the equation

$$
4 \pi T r_{h}-3 r_{h}^{2}-c_{1} r_{h}-c_{2}+\frac{\rho^{2}+h^{2}}{4 r_{h}^{2}}=0
$$

It is notice that $c_{1}$ offers an effective temperature correction, and make the solution complicated even at $T=0$. Although the $c_{2}$ is a constant playing a similar role as the momentum dissipation strength in ref. [77], the effect on the resistivity is quite different. The effect of $c_{1}$ and $c_{2}$ on $R_{x x}$ and $R_{x y}$ at zero temperature is presented in figure 2. For $c_{1}=0$ the $R_{x x}=0$ and $R_{x y}$ is constant, and is independent of $c_{2}$. For non-zero $c_{1}$, the $R_{x x}$ increases and saturates, while the $R_{x y}$ decreases to zero as $c_{1}$ becomes more negative. And a larger $\left|c_{2}\right|$ makes the surface steeper. In the following figures 3 to 5 we separately investigate the resistivities as functions of $\rho$ and $h$ under different $T$ and $c_{2}$. Because $c_{1}$ acts as an effective temperature modification, we set $c_{1}=-1$.

First, we consider zero temperature $T=0$, and $c_{2}=0$. No Mott-insulating behaviors and positive magneto-resistivities are observed in figure 3. In addition, non-zero charge density can suppress the resistivity as more charge carriers are introduced. In figure 5 the 

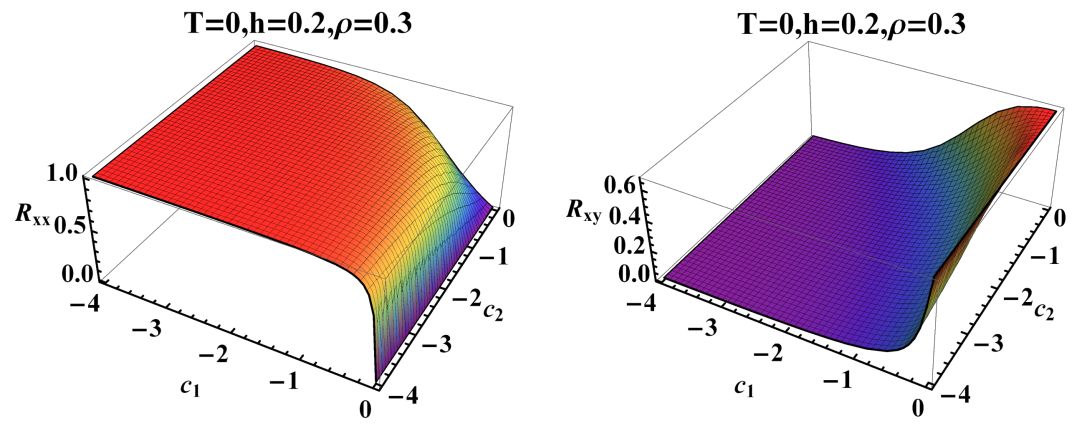

Figure 2. The influence of $c_{1}$ and $c_{2}$ on $R_{x x}$ and $R_{x y}$. We take $T=0$ and $\rho=0.3, h=0.2$ as an example.
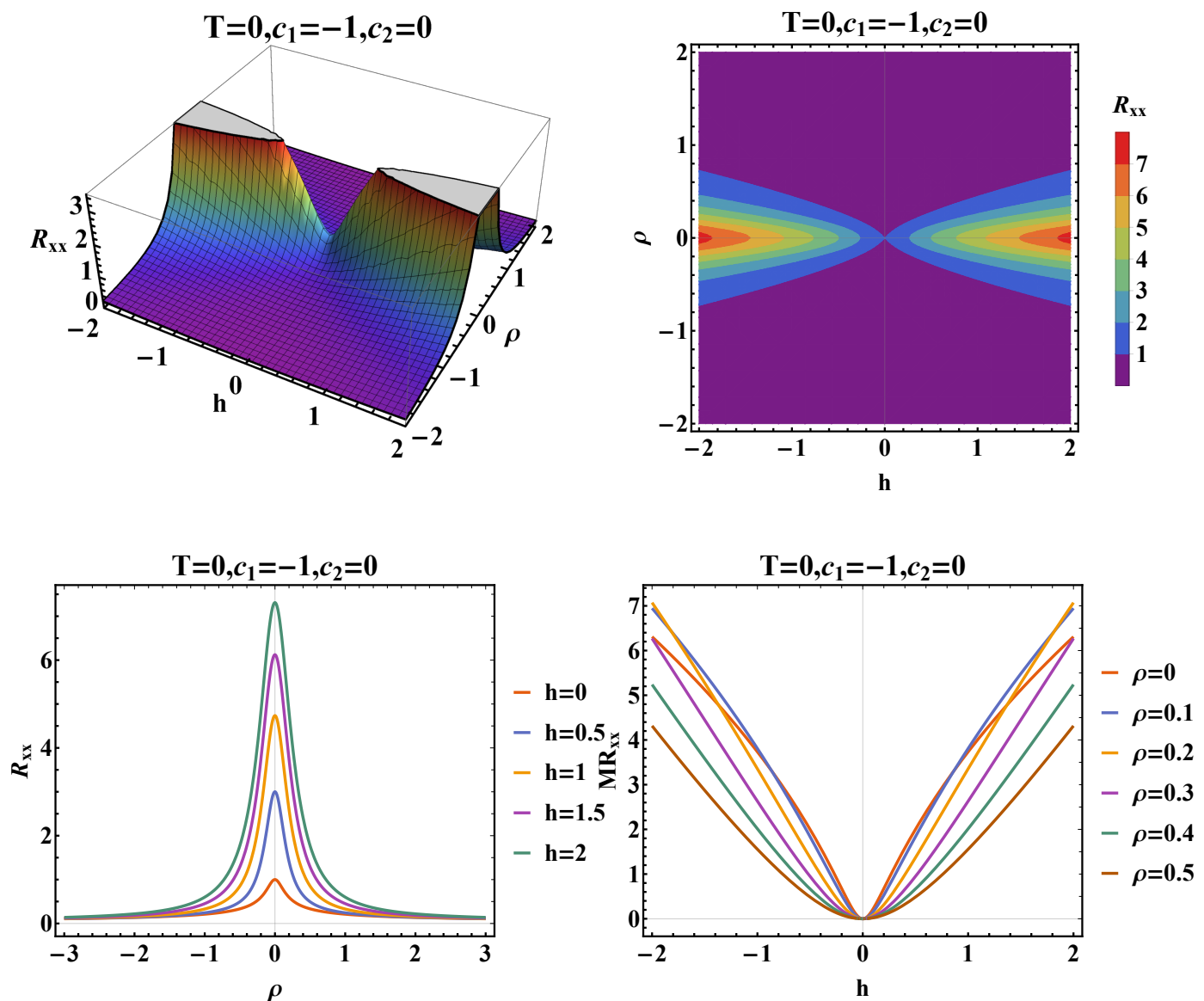

Figure 3. Upper left: $R_{x x}$ as a function of $\rho$ and $h$. Upper right: contour plot of $R_{x x}$. Lower left: $R_{x x}$ vs $\rho$ with $h=0,0.5,1,1.5,2$. Lower right: $M R_{x x}$ vs $h$ with $\rho=0,0.1,0.2,0.3,0.4,0.5$. We set $T=0, c_{1}=-1$, and $c_{2}=0$. 

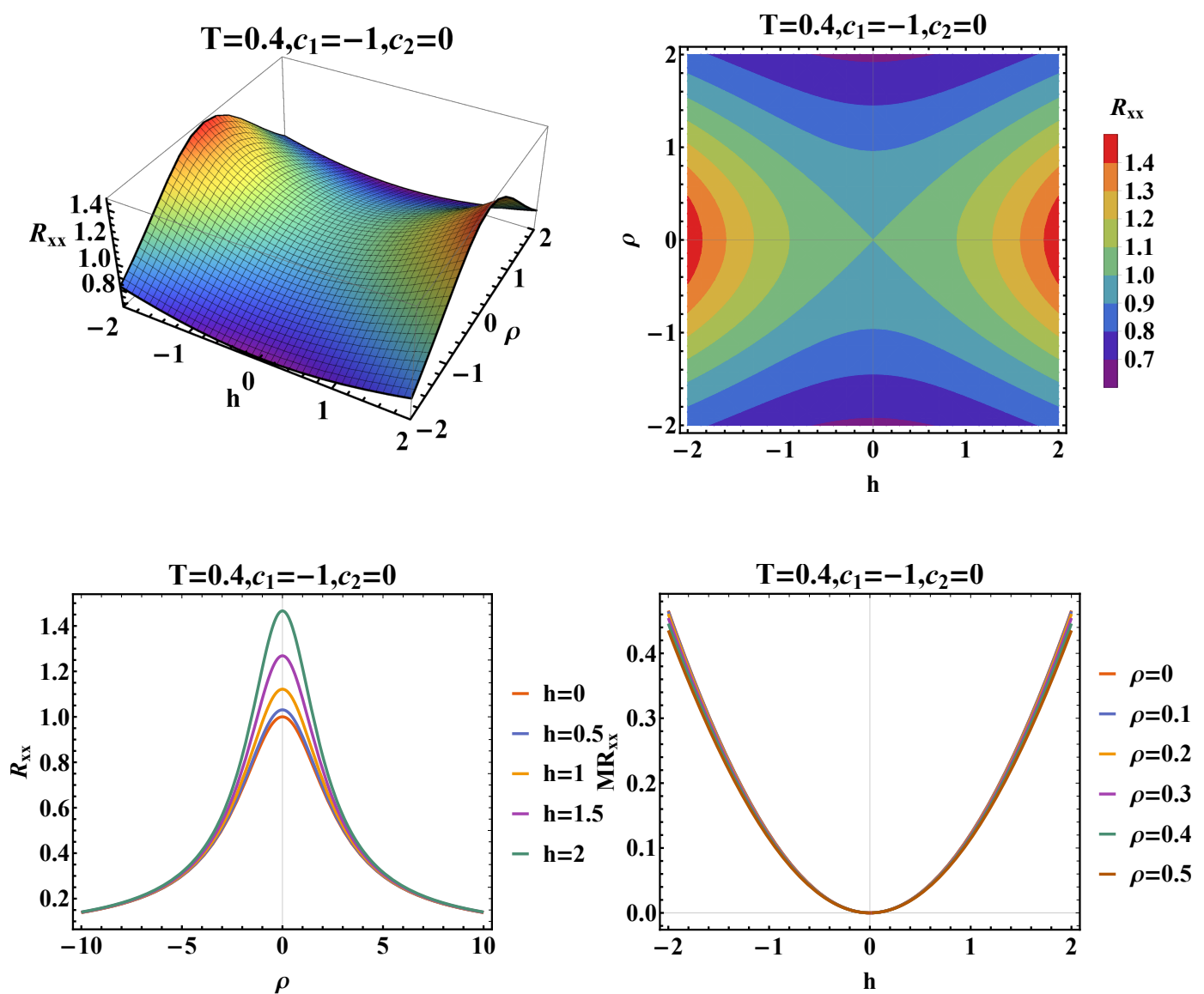

Figure 4. Upper left: $R_{x x}$ as a function of $\rho$ and $h$. Upper right: contour plot of $R_{x x}$. Lower left: $R_{x x}$ vs $\rho$ with $h=0,0.5,1,1.5,2$. Lower right: $M R_{x x}$ vs $h$ with $\rho=0,0.1,0.2,0.3,0.4,0.5$. We set $T=0.4, c_{1}=-1$, and $c_{2}=0$.

effect of $c_{2}$ at zero temperature is presented. The figures show similar feature as those in figure 4 . The larger $c_{2}$ also suppress the resistivity, while the influence is not so obvious as the temperature. All the pictures presented here do not exhibit the Mott-insulating behaviors and negative magneto-resistivities. Then the finite temperature situation with $T=0.4$ and $c_{2}=0$ is studied in figure 4 , where the saddle surface is relatively flatter than that in figure 3. And the magneto-resistivity is greatly suppressed by the temperature and the differences induced by charge density $\rho$ is smoothen out.

Then we present the dependence of $R_{x x}$ on $h / \rho$ and $T / \sqrt{\rho}$ for $c_{1}=-1$. The effect of different $c_{1}$ and $c_{2}$ can be deduced by change the temperature correspondingly, based on the analysis above. For $h<\rho$, figure 6 shows that the temperature dependence of $R_{x x}$ is monotonic, and corresponds to metallic behavior. For $h>\rho$, the $R_{x x}$ increases first and then decreases monotonically after reaching a maximum. The insulating behavior appears at high temperatures in this case. Moreover, if we take larger $c_{i}$, the metallic behaviors at $h>\rho$ would disappear and the $R_{x x}$ decreases monotonically with increasing temperature. So we come to the conclusion that increasing the magnetic field would induce 

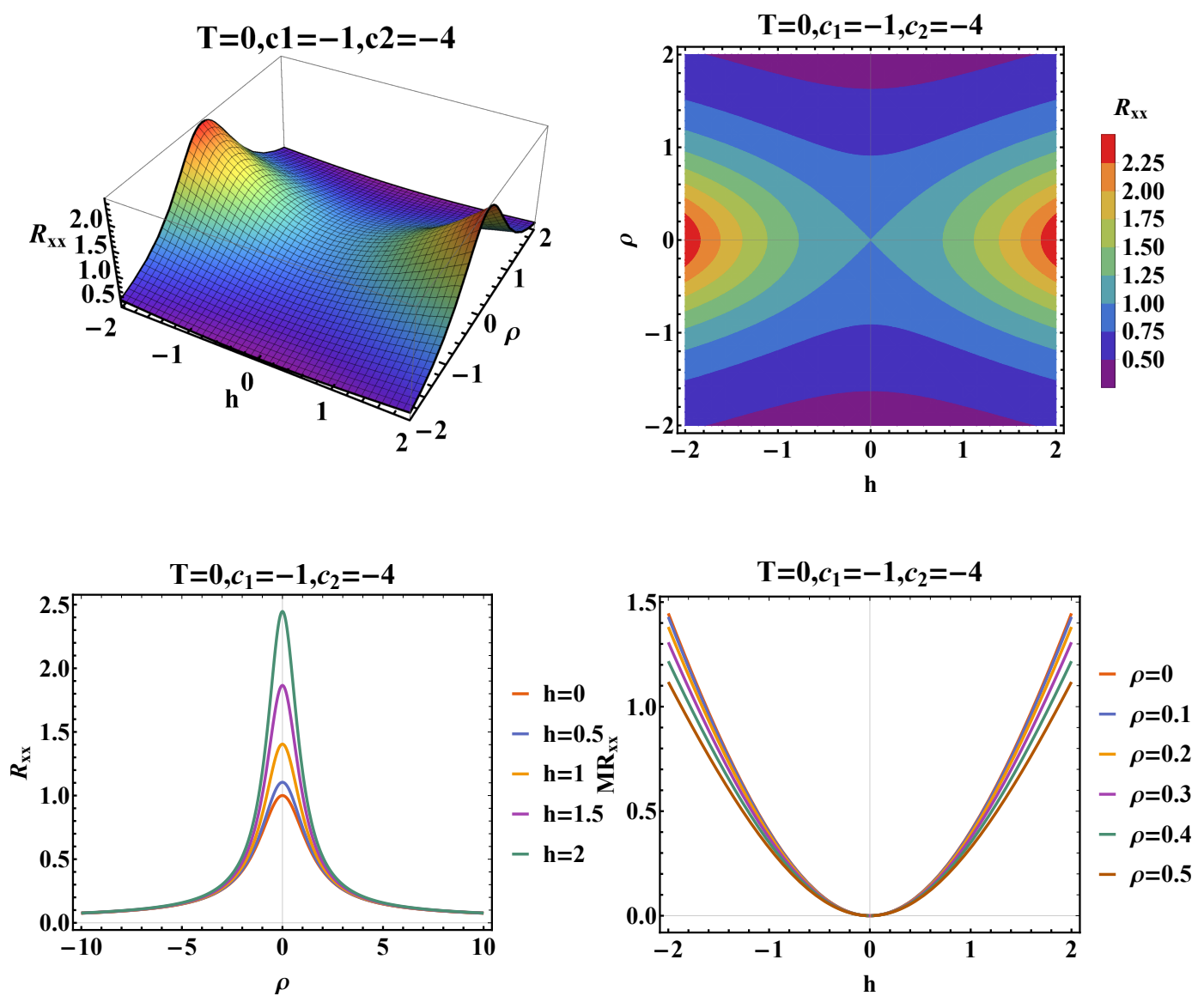

Figure 5. Upper left: $R_{x x}$ as a function of $\rho$ and $h$. Upper right: contour plot of $R_{x x}$. Lower left: $R_{x x}$ vs $\rho$ with $h=0,0.5,1,1.5,2$. Lower right: $M R_{x x}$ vs $h$ with $\rho=0,0.1,0.2,0.3,0.4,0.5$. We set $T=0, c_{1}=-1$, and $c_{2}=-4$.

a finite-temperature transition or crossover from metallic to insulating behavior. In the last sub-figure of figure 6 the influence of temperature on the magneto-resistivity is shown. As temperature increases, the magneto-resistivity is remarkably suppressed.

At the end of the discussion of Maxwell electrodynamics, the Hall angle $\theta_{H}$, defined as

$$
\theta_{H}=\arctan \frac{\sigma_{x y}}{\sigma_{x x}}
$$

is shown in figure 7. At zero temperature for large $h$ or $\rho$ the Hall angle get saturated, with the sign depend on those of $h$ or $\rho$. The surface is anti-symmetric under the transformation $(\rho, h) \rightarrow(\rho,-h)$ and $(\rho, h) \rightarrow(-\rho, h)$. As we expect, the temperature would evidently suppress the Hall angle.

\subsection{Maxwell-Chern-Simons electrodynamics}

The Lorentz and gauge invariance do not forbid the appearance of CP-violating ChernSimons $\theta$ term in the electrodynamics Lagrangian

$$
\mathcal{L}(s, p)=s+\theta p,
$$



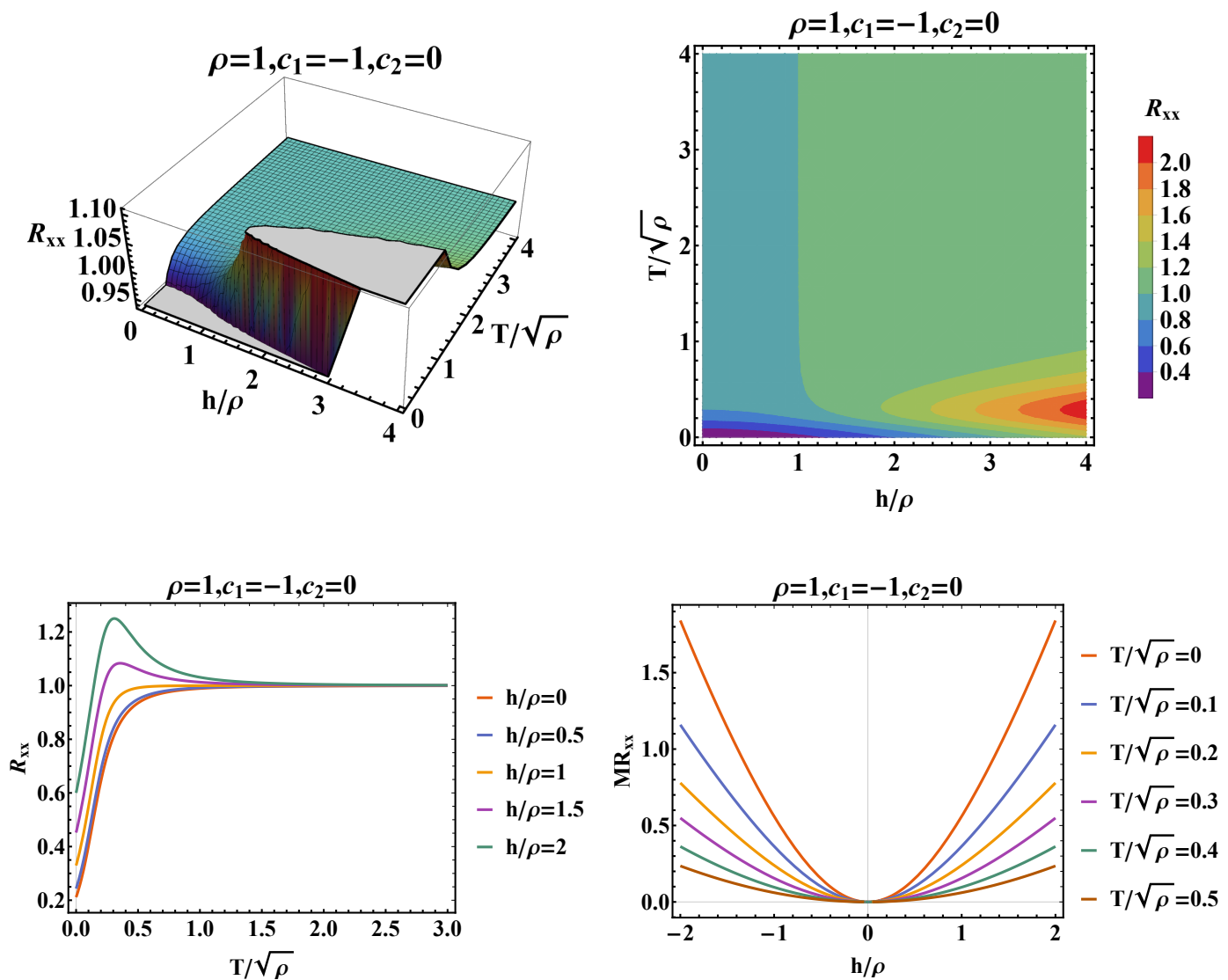

Figure 6. Upper left: $R_{x x}$ as a function of $h / \rho$ and $T / \sqrt{\rho}$. Upper right: contour plot of $R_{x x}$. Lower left: $R_{x x}$ vs $T / \sqrt{\rho}$ with $h / \rho=0,0.5,1,1.5,2$. Lower right: $M R_{x x}$ vs $h$ with $T / \sqrt{\rho}=$ $0,0.1,0.2,0.3,0.4,0.5$. We set $T=0, c_{1}=-1$, and $c_{2}=0$.
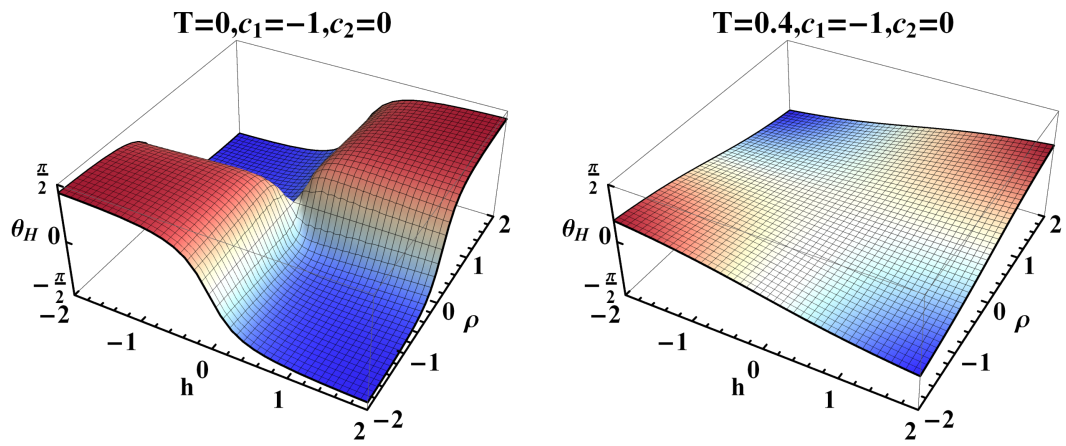

Figure 7. Hall angel at $T=0$ (left panel) and $T=0.4$ (right panel). $c_{1}=-1$ and $c_{2}=0$. 
and the Chern-Simons theory is of vital importance for the both integer and fractional quantum Hall effects in condensed matter physics [78-81]. And the value of $\theta$ can be related to the Hall conductivity in the unit of $e^{2} / \hbar$. After taking the Chern-Simons term into consideration, eq. (3.4) gives

$$
A_{t}^{\prime}\left(r_{h}\right)=\frac{\rho+\theta h}{r_{h}^{2}}
$$

and the eq. (2.13) becomes

$$
4 \pi T r_{h}-3 r_{h}^{2}-c_{1} r_{h}-c_{2}+\frac{\left(1+\theta^{2}\right) h^{2}+2 \theta h \rho+\rho^{2}}{4 r_{h}^{2}}=0 .
$$

We then reinvestigate the dependence of DC resistivity $R_{x x}$ on $\rho$ and $h$. As one expects, the similar saddle surface to that of Maxwell electrodynamics is found, and the reflection asymmetry and central symmetry due to the $\theta$ term is shown in the upper panel of figure 8 . The more surprising things are the appearance of Mott-insulating behavior $\partial_{|\rho|} R_{x x}>0$ and negative magneto-resistivity $M R_{x x}<0$. At zero field $R_{x x}$ is the even function of $\rho$, and as $|\rho|$ increases, the value of $R_{x x}$ increases and reaches a maximum, showing the feature of Mott-insulating behavior, and then decreases monotonically. While for finite magnetic field, the reflection symmetry for positive and negative $\rho$ is broken. Along the positive $\rho$ direction the behavior of $R_{x x}$ is similar, however, for negative $\rho$ it is more complicated. As $|\rho|$ increases, the value of $R_{x x}$ decreases and reaches a minimum, and then shares the similar behaviors as the positive $\rho$ case. We then further study how the value of $\theta$ affect the Mottinsulating behavior in figure 9. To clearly show the region $\partial_{|\rho|} R_{x x}>0$ we set other region's value to be zero. Similar results have been obtained as in ref. [77]. At zero field there is no Mott-insulating behavior for $\theta=1$. A larger $\theta$ makes it possible even with $h=0$. On the other hand, the Mott-insulating region becomes larger but $\partial_{|\rho|} R_{x x}$ becomes smaller as $\theta$ increases. The negative magneto-resistivity revealed in figure 8 is also shown in the $\rho-h$ parameter plane in figure 10, from which we can see that the negative magneto-resistivity emerges in the finite interval of $h / \rho$ of about $[-1.2,0]$. The positive magneto-resistivity region's value is set to be zero as well. At larger magnetic field, the negative magnetoresistivity phenomenon disappears, and the magneto-resistivity increases almost linearly with $h$. For zero density the negative magneto-resistivity does not appear. Finally we show the behavior of Hall resistivity $R_{x y}$ and Hall conductivity $\sigma_{x y}$ in figure 11 . The negative Hall resistivity comes from the negative transverse conductivity $\sigma_{x y}$ depending on the direction of magnetic field $h$ and the type of charge carriers. From the figure we see that at zero field the transverse conductivity $\sigma_{x y}$ is exactly $-\theta$ in regardless of charge density $\rho$, which agrees with the scenario in quantum Hall physics $[42,80]$. At finite magnetic field, for positive $\rho$ the Hall conductivity will decreases to zero, and then changes its sign, while for negative $\rho$ the Hall conductivity remains negative. And we can also study the impact of magnetic field on the Hall conductivity with fixed $\rho$. All the transverse conductivities for various $\rho$ as strong field tend to be zero as the result of localization. For positive $\rho$, in the positive magnetic field, the Hall conductivity decreases to zero, then changes its sign and increases to a maximum, and finally decreases monotonically to zero from the positive 

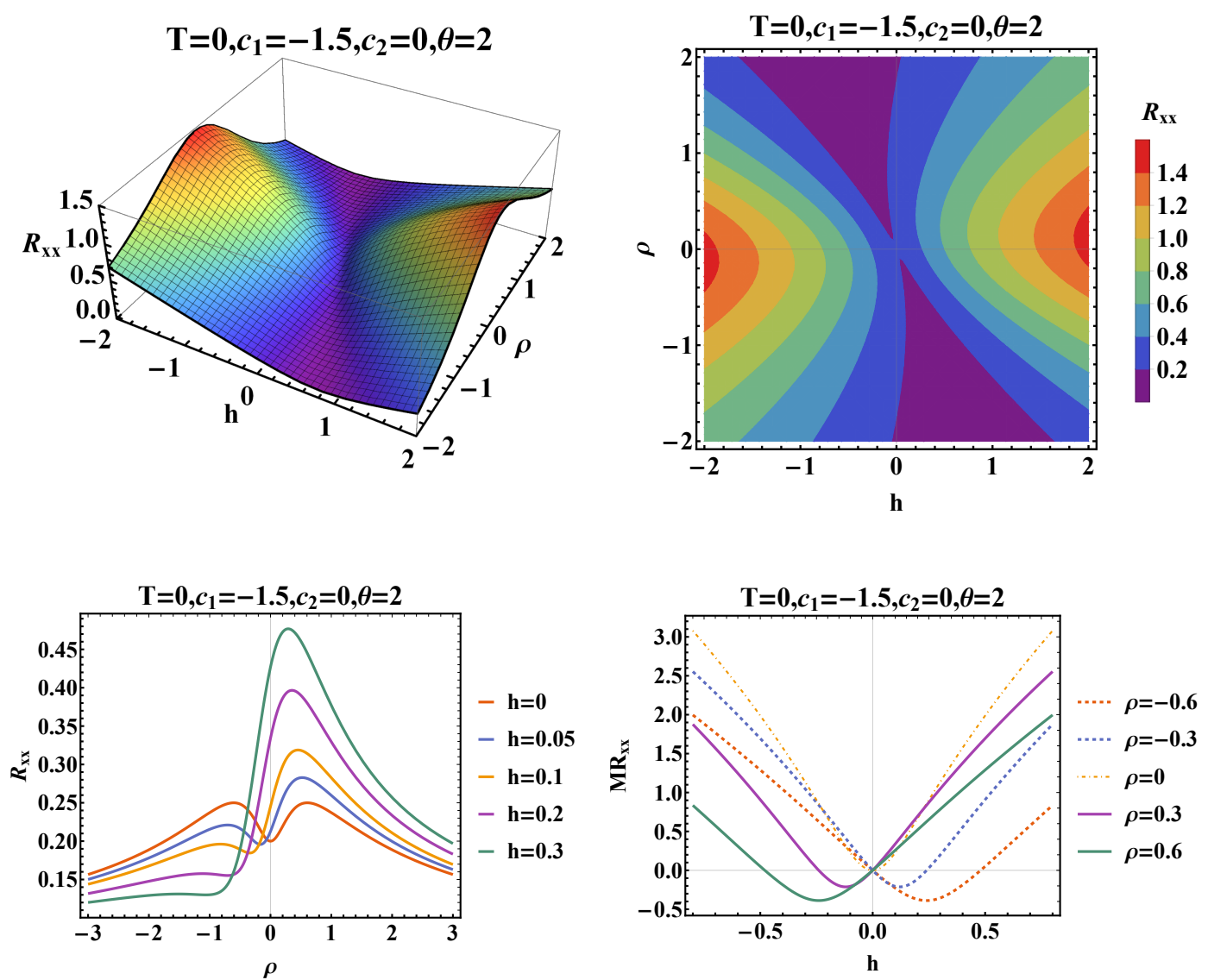

Figure 8. Upper left: $R_{x x}$ as a function of $h$ and $\rho$. Upper right: contour plot of $R_{x x}$. Lower left: $R_{x x}$ vs $\rho$ with $h=0,0.05,0.1,0.2,0.3$. Lower right: $M R_{x x}$ vs $h$ with $\rho=-0.6,-0.3,0,0.3,0.6$. We set $T=0, c_{1}=-1.5, c_{2}=0$ and $\theta=2$.
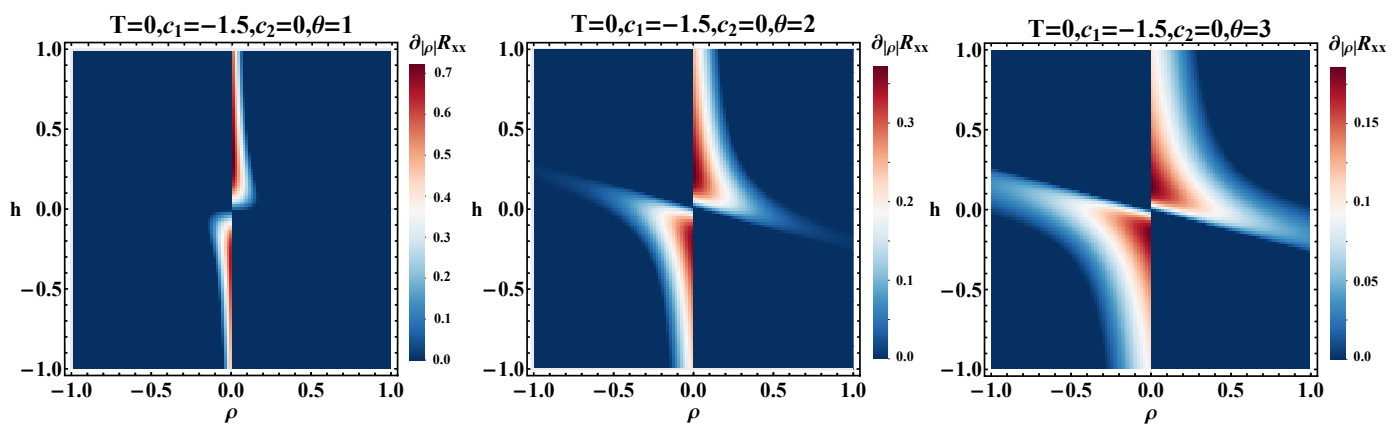

Figure 9. Mott-insulating region for $\theta=1,2,3$ in the parameter plane for Maxwell-Chern-Simons electrodynamics at zero temperature. We set $c_{1}=-1.5$ and $c_{2}=0$. 


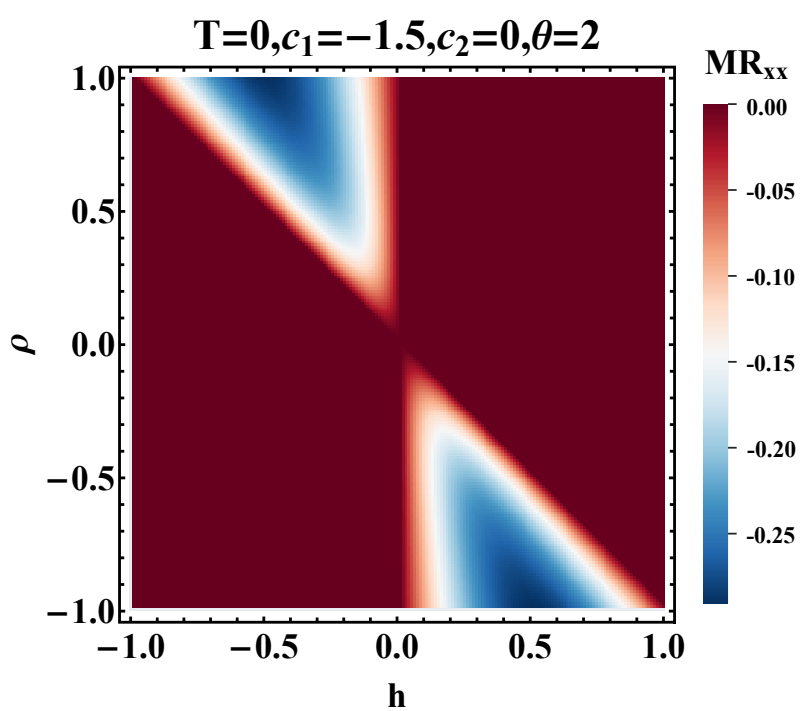

Figure 10. Negative magneto-resistivity region for $\theta=3$ in the parameter plane for MaxwellChern-Simons electrodynamics at zero temperature. We set $c_{1}=-1.5$ and $c_{2}=0$.

side. And in the negative magnetic field, the Hall conductivity increases to a negative maximum, and then decreases to zero from the negative side. The case of negative $\rho$ can be known by simply making transformation $(\rho, h) \rightarrow(-\rho,-h)$ in the discussion above.

\subsection{Born-Infeld electrodynamics}

The Born-Infeld electrodynamics is described by a square-root Lagrangian $[82,83]$

$$
\mathcal{L}(s, p)=\frac{1}{a}\left(1-\sqrt{1-2 a s-a^{2} p^{2}}\right),
$$

where the coupling parameter $a=\left(2 \pi \alpha^{\prime}\right)^{2}$ relates to the Regge slope $\alpha^{\prime}$. It is believed that such a NLED governs the dynamics of electromagnetic fields on D-branes. If we take the zero-slope limit $\alpha^{\prime} \rightarrow 0$, the Maxwell Lagrangian is recovered

$$
\mathcal{L}(s, p)=s+\mathcal{O}(a)
$$

The Born-Infeld electrodynamics takes the advantage of eliminating the divergence of electrostatic self-energy and incorporating maximal electric field strength [84]. This can be seen from the solution of eq. (3.4)

$$
A_{t}^{\prime}(r)=\frac{\rho}{\sqrt{a\left(\rho^{2}+h^{2}\right)+r^{4}}},
$$

which is finite when $r \rightarrow 0$. The $r_{h}$ is solved form

$$
4 \pi r_{h} T-3 r_{h}^{2}-c_{1} r_{h}-c_{2}+\frac{\rho^{2}}{2 \sqrt{a\left(h^{2}+\rho^{2}\right)+r_{h}^{4}}}+\frac{1}{2 a}\left(\sqrt{\frac{\left(a h^{2}+r_{h}^{4}\right)^{2}}{a\left(h^{2}+\rho^{2}\right)+r_{h}^{4}}}-r_{h}^{2}\right)=0
$$



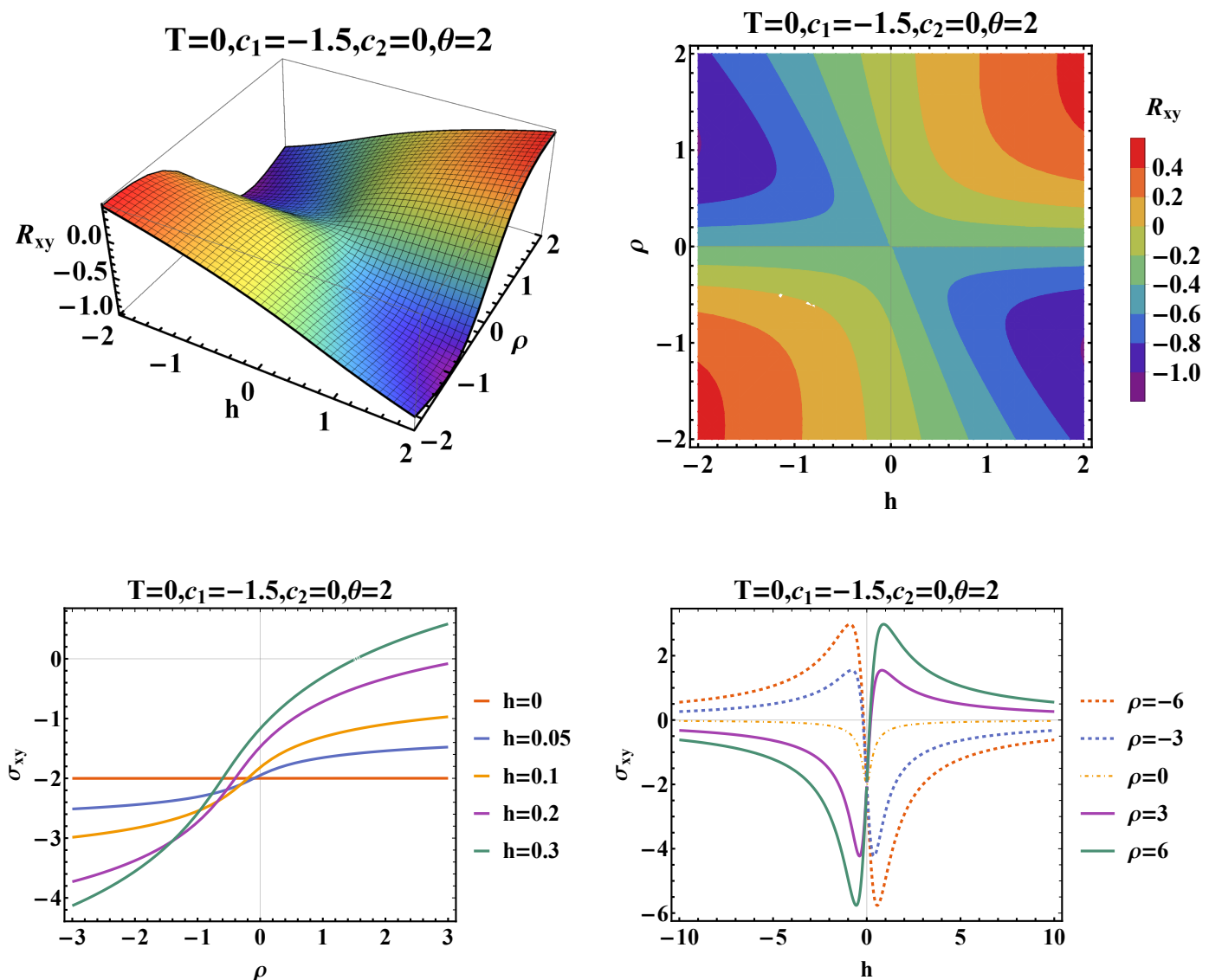

Figure 11. Upper left: $R_{x y}$ as a function of $h$ and $\rho$. Upper right: contour plot of $R_{x y}$. Lower left: $\sigma_{x y}$ vs $\rho$ with $h=0,0.05,0.1,0.2,0.3$. Lower right: $\sigma_{x y}$ vs $h$ with $\rho=-6,-3,0,3,6$. We set $T=0, c_{1}=-1.5, c_{2}=0$ and $\theta=2$.

The DC resistivity is then studied in the framework of Born-Infeld electrodynamics and the results are shown in figure 13. For positive $a$ the behavior of $R_{x x}$ is similar to the Maxwell case [69, 77], and the negative $a$ can bring in more interesting phenomenon. However, for negative $a$ the eq. (4.9) suffers a singularity $r=r_{s} \equiv \sqrt[1 / 4]{|a|\left(h^{2}+\rho^{2}\right)}$, and the physical solution requires that $r_{h}>r_{s}$, setting an upper bound for $h^{2}+\rho^{2}$ [77]. We then present two cases of $a=-0.4$ and $a=-1$, respectively. For $a=-0.4$, We see the saddle surface is similar to the previous results, and there is no Mott-insulating behaviors and negative magneto-resistivities. However, if one increases the absolute value of the negative $a$, the region admitting physical solution for $R_{x x}$ shrinks and is truncated at the upper bound of $h^{2}+\rho^{2}$. For zero and finite small magnetic field, the Mott-insulating behavior is absent, while for a larger field the Mott-insulating behavior emerges. And for a relatively small $\rho$, the negative magneto-resistivity is present, and increasing $\rho$ can destroy it. To expose the role of $a$ we expand the interaction between electrons to the first order [77]

$$
F(a)=\rho A_{t}^{\prime}(r) \sim \frac{\rho^{2}}{r^{2}}\left[1-\frac{a}{2 r^{4}}\left(h^{2}+\rho^{2}\right)\right]+\mathcal{O}\left(a^{2}\right)
$$



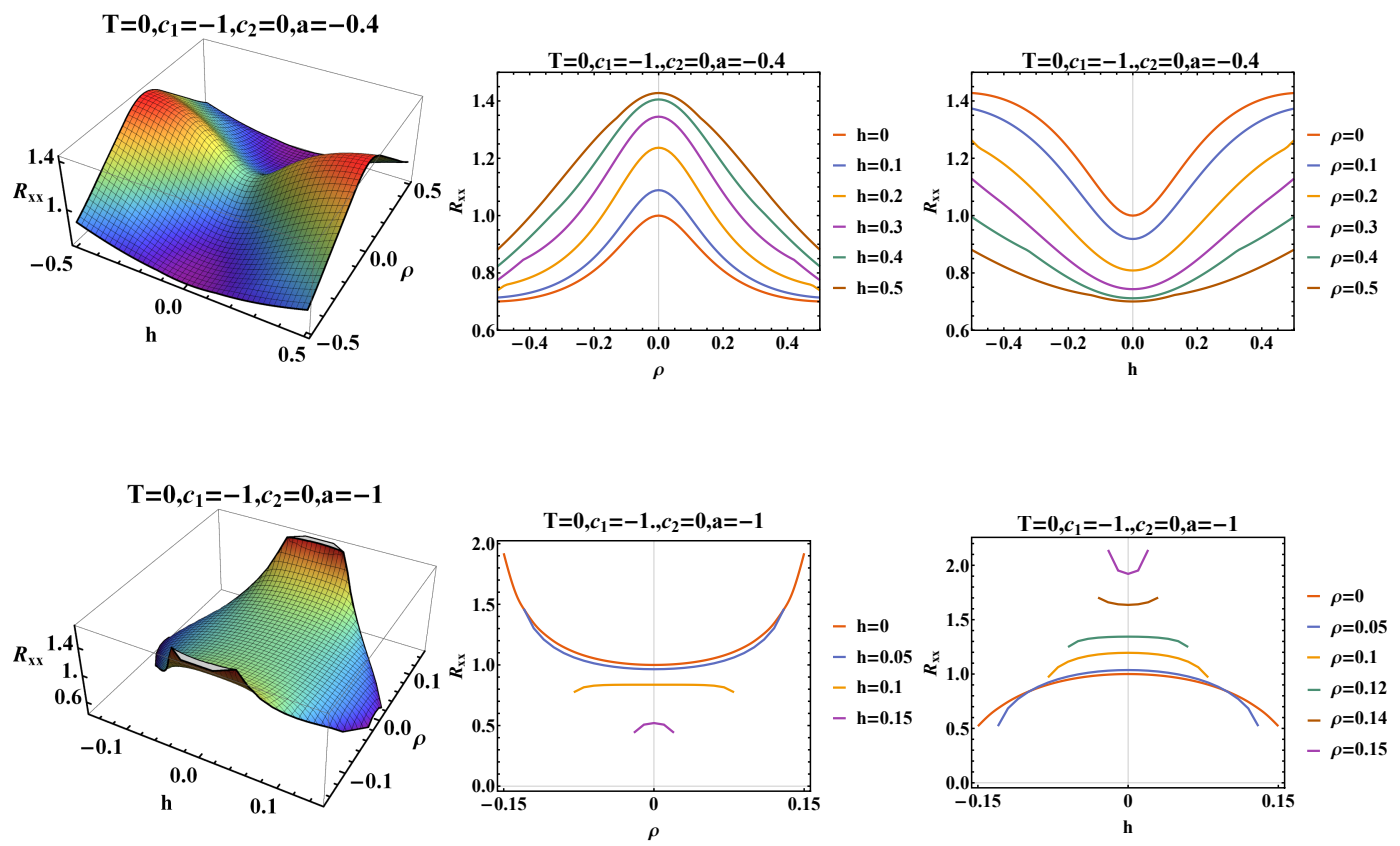

Figure 12. $R_{x x}$ as a function of $h$ and $\rho$ and some intersection curves for fixed $h$ and $\rho$ for $a=-0.4$ (upper panel) and $a=-1$ (lower panel). The surface ends at some place where $h$ and $\rho$ touch the upper bound.

in which the leading order is the familiar Coulomb interaction, and the nonlinearity parameter serves as an effective modification. A positive $a$ suppress the interaction and we do not expect different phenomenon compared with the Maxwell electrodynamics. However, for a negative $a$ the interaction is enhanced at $r_{h}$

$$
\frac{F(a)}{F(0)}=1+\frac{1}{2}\left(\frac{r_{s}}{r_{h}}\right)^{4}
$$

and we expect that the NLED model with negative $a$ can grasp some features of strongly correlated systems. Besides, in the leading order expansion eq. (4.8) the Chern-Simons term does not appear, and can be deduced from eq. (3.19) that the system will not exhibit Mott-insulating behavior and negative magneto-resistivity. Thus we conclude that the Born-Infeld electrodynamics provides a new mechanism different from the Chern-Simons theory to give rise to the Mott-insulating behavior and negative magneto-resistivity, and the temperature can induce a transition at finite temperature.

One can also consider another kind of construction of square-root Lagrangian [84]

$$
\mathcal{L}(s, p)=\frac{1}{a}(1-\sqrt{1-2 a s}),
$$

which has the same leading order expansion as eq. (4.8), and will re-derive the results of Born-Infeld electrodynamics in the presence of zero field. The solution of eq. (3.4) gives

$$
A_{t}^{\prime}(r)=\frac{\rho}{r^{2}} \sqrt{\frac{a h^{2}+r^{4}}{a \rho^{2}+r^{4}}},
$$



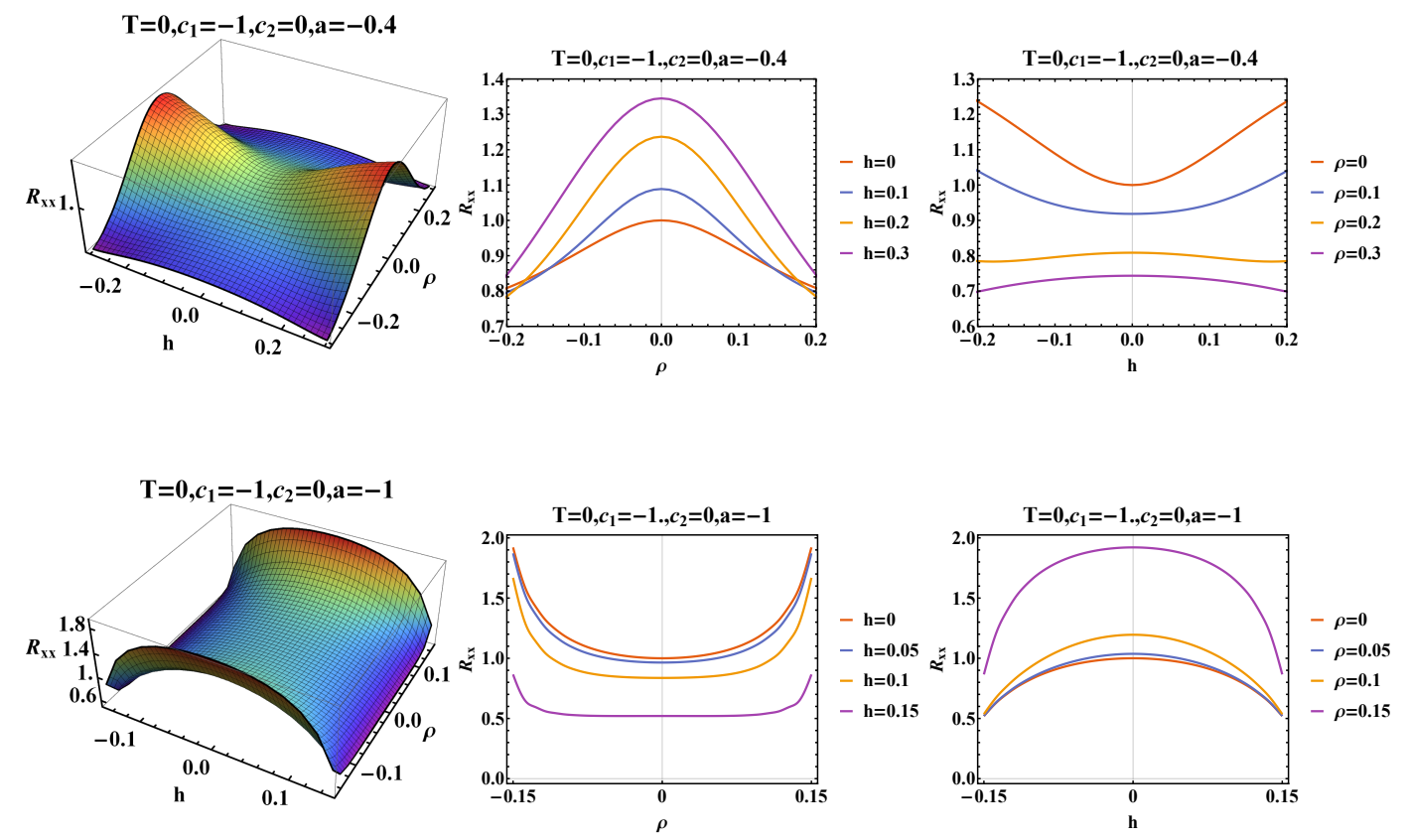

Figure 13. $R_{x y}$ as a function of $h$ and $\rho$ and some intersection curves for fixed $h$ and $\rho$ for $a=-0.4$ (upper panel) and $a=-1$ (lower panel).

which can be deduced that the effective interaction is

$$
F=\rho A_{t}^{\prime}(r) \sim \frac{\rho^{2}}{r^{2}}\left[1-\frac{a}{2 r^{4}}\left(\rho^{2}-h^{2}\right)\right]+\mathcal{O}\left(a^{2}\right) .
$$

The radius equation is

$$
4 \pi r_{h} T-3 r_{h}^{2}-c_{1} r_{h}-c_{2}+\frac{h^{2}}{2 r_{h}^{2}}+\frac{r_{h}^{2}}{2 a}\left(\sqrt{\frac{a \rho^{2}+r_{h}^{4}}{a h^{2}+r_{h}^{4}}}-1\right)=0 .
$$

We re-check the $R_{x x}$ in the square-root electrodynamics for negative $a$. For $a=-0.4$ the saddle surface is similar to the previous results, and both the zero field and finite field no Mott insulating behaviors are shown. And for fixed $\rho$, it is surprising that for larger $\rho$, the transition from positive magneto-resistivity to negative magneto-resistivity is observed for about $\rho>h$. We then studied the case of larger negative $a$. To our surprise, the saddle surface changes its direction and looks as if it rotates $90^{\circ}$. We find that the Mott-insulating behavior appears and becomes significant at large $\rho$, and negative magneto-resistivity is observed for various $\rho$.

\section{Conclusion}

In this work the black brane solution of four-dimensional massive gravity with backreacted NLED is obtained, and with the dictionary of gauge/gravity duality, the transport properties of the strongly correlated systems in the presence of finite magnetic field in $2+1$ dimensional boundary is studied. In our holographic setup the bulk geometry and NLED 


\begin{tabular}{|c|c|c|c|c|}
\hline & Lagrangian & Parameter & Mott-insulating behavior & Negative magneto-resistivity \\
\hline Maxwell & $s$ & & No & No \\
\hline $\begin{array}{l}\text { Maxwell- } \\
\text { Chern- } \\
\text { Simons }\end{array}$ & $s+\theta p$ & $\theta$ & See figure 9 & See figure 10 \\
\hline \multirow[b]{3}{*}{ Born-Infeld } & \multirow[b]{3}{*}{$\frac{1}{a}\left(1-\sqrt{1-2 a s-a^{2} p^{2}}\right)$} & $a>0$ & No & No \\
\hline & & $a=-0.4$ & No & No \\
\hline & & $a=-1$ & $\begin{array}{c}\text { Finite } h \text { and small } \rho . \\
\text { See figure } 12\end{array}$ & $\begin{array}{c}\text { Finite } h \text { and small } \rho . \\
\text { See figure } 12\end{array}$ \\
\hline \multirow{3}{*}{ Square } & \multirow{3}{*}{$\frac{1}{a}(1-\sqrt{1-2 a s})$} & $a>0$ & No & No \\
\hline & & $a=-0.4$ & No & Larger $\rho$. See figure 13 \\
\hline & & $a=-1$ & Larger $\rho$. See figure 13 & Yes \\
\hline
\end{tabular}

Table 1. Conclusion of Mott-insulating behavior and negative magneto-resistivity for various NLED models at zero temperature $T=0$.

field are perturbed, and the DC conductivities are obtained in the linear response regime. Then some general properties are obtained in various limits, which agrees well with the previous work. To make it concrete, we present the study of the conventional Maxwell electrodynamics, the topological non-trivial Maxwell-Chern-Simons electrodynamics, and the Born-Infeld electrodynamics with string-theoretical correction taken into consideration. We concentrate on two interesting phenomena, i.e., the Mott-insulating behavior and negative magneto-resistivity, and results at zero temperature are summarized in table 1.

We briefly revisit the various limits for Born-Infeld electrodynamics. In the massless limit, the $\sigma_{x x}$ has a lower bound controlled by $r_{s}$, which is affected by the NLED parameter $a$. In the massive limit, For the Maxwell-Chern-Simons electrodynamics, the DC conductivities is $\sigma_{x x}=1$ and $\sigma_{x y}=-\theta$. While for the Born-Infeld electrodynamics, we have

$$
\begin{aligned}
\sigma_{x x} & =\frac{r_{h}^{2} \sqrt{r_{h}^{4}+a\left(h^{2}+\rho^{2}\right)}}{r_{h}^{4}+a h^{2}}, \\
\sigma_{x y} & =\frac{a h \rho}{r_{h}^{4}+a h^{2}} .
\end{aligned}
$$

The $\sigma_{x x}$ is suppressed for negative $a$ and will vanishes at $r_{s}$. Besides, the negative $a$ implies the Mott-insulating behavior. And the Hall conductivity does not vanish as a result of non-zero $a$. For the zero field or charge density limit, the Hall conductivity vanishes in both cases. And the longitudinal conductivity has the form

$$
\begin{aligned}
& \sigma_{x x}(h=0)=\left(1-\frac{a \rho^{2}}{a \rho^{2}+r_{h}^{4}}\right)^{-1 / 2}-\frac{\rho^{2}}{\alpha m^{2} c_{1} r_{h}^{3}}, \\
& \sigma_{x x}^{-1}(\rho=0)=\left(1+\frac{a h^{2}}{r_{h}^{4}}\right)^{-1 / 2}-\frac{h^{2}}{\alpha m^{2} c_{1} r_{h}^{3}} .
\end{aligned}
$$

We see that when reaching $r_{s}$, the square root part is removed from the formula, leaving the rest part with a quadratic dependence on $h$ or $\rho$. 
The massive gravity coupling parameters' influence on the in-plane resistivity is compared with the temperature, where we find the $c_{1}$ behaves as the effective temperature correction, and non-zero one can lead to the DC conductivity. While the $c_{2}$ has less significant effect on $R_{x x}$ than $c_{1}$. Moreover, the dependence on $\rho$ and $h$ is shown and we find the field can induce the metal-insulator transition or Mott-insulating behavior and negative magneto-resistivity. Two different mechanism, the Chern-Simons term, and the negative nonlinearity parameter are proved that can give rise to Mott-insulating behavior and negative magneto-resistivity. The fact that the nonlinearity construction can result in the negative magneto-resistivity and Mott insulating behavior provides a different mechanism without CP-violation construction in Chern-Simons term. Besides, the effective interaction extracted from the NLED Lagrangian can qualitatively account for the presence of Mott insulating behavior. However, there are also some shortcomings. To avoid the appearance of naked singularity, we have to make some restriction on the radius of event horizon, which sets an upper bound for the magnetic field and charge density for the black hole, and the physical solution space shrinks. In addition, how the nonlinearities brings in the negative magneto-resistivity remains to be explored. We hope our work could explain some experimental phenomenon in strongly correlated systems.

\section{A Consistency of metric ansatz}

The reparametrisation freedom for the radial coordinate is not enough to fix both $g_{t t}=$ $-1 / g_{r r}$ and $g_{x x}=g_{y y}=r^{2}$ at the same time. To test the validity of eq. (2.8a), we write the metric as [71]:

$$
\begin{aligned}
\mathrm{d} s^{2} & =-N(r)^{2} f(r) \mathrm{d} t^{2}+\frac{\mathrm{d} r^{2}}{f(r)}+r^{2}\left(\mathrm{~d} x^{2}+\mathrm{d} y^{2}\right), \\
A & =A_{t}(r) \mathrm{d} t+\frac{h}{2}(x \mathrm{~d} y-y \mathrm{~d} x), \\
f_{\mu \nu} & =\operatorname{diag}\left(0,0, \alpha^{2}, \alpha^{2}\right)
\end{aligned}
$$

where $N(r)$ is a scale function of $r$ and will be proved to be a constant, and thus can be absorbed into the coordinate $t$. With the reference metric $f_{\mu \nu}$ we have

$$
\mathcal{U}_{1}=2 \alpha / r, \mathcal{U}_{2}=2 \alpha^{2} / r^{2}, \mathcal{U}_{3}=\mathcal{U}_{4}=0
$$

and the non-vanishing $G^{\mu \nu}$ in eq. (2.7) in the main text are

$$
\begin{aligned}
G^{r t} & =\frac{\partial \mathcal{L}}{\partial p} \frac{h}{N(r) r^{2}}-\frac{\partial \mathcal{L}}{\partial s} \frac{A_{t}^{\prime}(r)}{N(r)^{2}} \\
G^{x y} & =\frac{\partial \mathcal{L}}{\partial s} \frac{h}{r^{4}}+\frac{\partial \mathcal{L}}{\partial p} \frac{A_{t}^{\prime}(r)}{N(r) r^{2}}
\end{aligned}
$$


We now consider the equations of motion for $t$ and $r$ component:

$$
\begin{aligned}
& r f^{\prime}(r)+f(r)-3 r^{2}=c_{1} \alpha m^{2} r+c_{2} \alpha^{2} m^{2}+\frac{r^{2}}{2}\left(A_{t}^{\prime}(r) G^{r t}+\mathcal{L}(s, p)\right), \\
& r f^{\prime}(r)+f(r)-3 r^{2}=c_{1} \alpha m^{2} r+c_{2} \alpha^{2} m^{2}+\frac{r^{2}}{2}\left(A_{t}^{\prime}(r) G^{r t}+\mathcal{L}(s, p)\right)+4 r f(r) N(r) N^{\prime}(r) .
\end{aligned}
$$

From the equations of motion above we see that eq. (A.4a) is the same as eq. (2.10a) in the main text for the $t$ component, and eq. (A.4b) has an extra term $4 r f(r) N(r) N^{\prime}(r)$. We conclude that $4 r f(r) N(r) N^{\prime}(r)=0$, and the physical solution is that $N^{\prime}(r)=0$. Therefore, $N(r)$ is a constant and can be eliminated by rescaling the $t$ coordinate, and we come to the metric in eq. (2.8a) in the math text.

\section{Acknowledgments}

We are grateful to thank Peng Wang and Bo Ning for useful discussions. This work is supported by NSFC (Grant No. 11947408 and No. 12047573).

Open Access. This article is distributed under the terms of the Creative Commons Attribution License (CC-BY 4.0), which permits any use, distribution and reproduction in any medium, provided the original author(s) and source are credited.

\section{References}

[1] T. Banks, W. Fischler, S.H. Shenker and L. Susskind, $M$ theory as a matrix model: A Conjecture, Phys. Rev. D 55 (1997) 5112 [hep-th/9610043] [INSPIRE].

[2] J.M. Maldacena, The Large $N$ limit of superconformal field theories and supergravity, Int. J. Theor. Phys. 38 (1999) 1113 [hep-th/9711200] [INSPIRE].

[3] E. Witten, Anti-de Sitter space and holography, Adv. Theor. Math. Phys. 2 (1998) 253 [hep-th/9802150] [INSPIRE].

[4] M. Ammon and J. Erdmenger, Gauge/Gravity Duality: Foundations and Applications, Cambridge University Press (2015) [DOI].

[5] E. Papantonopoulos, ed., From gravity to thermal gauge theories: The AdS/CFT correspondence, Lect. Notes Phys. 828 (2011) 1 [INSPIRE].

[6] M. Natsuume, AdS/CFT Duality User Guide, Lect. Notes Phys. 903 (2015) 1 [arXiv: 1409.3575] [INSPIRE].

[7] G. Policastro, D.T. Son and A.O. Starinets, The Shear viscosity of strongly coupled $N=4$ supersymmetric Yang-Mills plasma, Phys. Rev. Lett. 87 (2001) 081601 [hep-th/0104066] [INSPIRE].

[8] A. Buchel and J.T. Liu, Universality of the shear viscosity in supergravity, Phys. Rev. Lett. 93 (2004) 090602 [hep-th/0311175] [INSPIRE].

[9] P. Kovtun, D.T. Son and A.O. Starinets, Viscosity in strongly interacting quantum field theories from black hole physics, Phys. Rev. Lett. 94 (2005) 111601 [hep-th/0405231] [INSPIRE]. 
[10] P. Benincasa and A. Buchel, Transport properties of $N=4$ supersymmetric Yang-Mills theory at finite coupling, JHEP 01 (2006) 103 [hep-th/0510041] [INSPIRE].

[11] D.T. Son and A.O. Starinets, Hydrodynamics of r-charged black holes, JHEP 03 (2006) 052 [hep-th/0601157] [INSPIRE].

[12] D.T. Son and A.O. Starinets, Viscosity, Black Holes, and Quantum Field Theory, Ann. Rev. Nucl. Part. Sci. 57 (2007) 95 [arXiv:0704.0240] [INSPIRE].

[13] P. Kovtun, Lectures on hydrodynamic fluctuations in relativistic theories, J. Phys. A 45 (2012) 473001 [arXiv:1205.5040] [INSPIRE].

[14] M. Rangamani, Gravity and Hydrodynamics: Lectures on the fluid-gravity correspondence, Class. Quant. Grav. 26 (2009) 224003 [arXiv:0905.4352] [INSPIRE].

[15] A. Adams and E. Silverstein, Closed string tachyons, AdS/CFT, and large $N$ QCD, Phys. Rev. D 64 (2001) 086001 [hep-th/0103220] [INSPIRE].

[16] S.J. Brodsky and G.F. de Téramond, Light-front hadron dynamics and AdS/CFT correspondence, Phys. Lett. B 582 (2004) 211 [hep-th/0310227] [INSPIRE].

[17] G.F. de Teramond and S.J. Brodsky, Hadronic spectrum of a holographic dual of QCD, Phys. Rev. Lett. 94 (2005) 201601 [hep-th/0501022] [INSPIRE].

[18] G.F. de Teramond and S.J. Brodsky, Light-Front Holography: A First Approximation to QCD, Phys. Rev. Lett. 102 (2009) 081601 [arXiv:0809.4899] [INSPIRE].

[19] J. Erlich, E. Katz, D.T. Son and M.A. Stephanov, QCD and a holographic model of hadrons, Phys. Rev. Lett. 95 (2005) 261602 [hep-ph/0501128] [INSPIRE].

[20] L. Da Rold and A. Pomarol, Chiral symmetry breaking from five dimensional spaces, Nucl. Phys. B 721 (2005) 79 [hep-ph/0501218] [INSPIRE].

[21] A.V. Zayakin, QCD Vacuum Properties in a Magnetic Field from AdS/CFT: Chiral Condensate and Goldstone Mass, JHEP 07 (2008) 116 [arXiv:0807.2917] [INSPIRE].

[22] J.D. Edelstein, J.P. Shock and D. Zoakos, The AdS/CFT Correspondence and Non-perturbative QCD, AIP Conf. Proc. 1116 (2009) 265 [arXiv:0901.2534] [INSPIRE].

[23] U. Gürsoy, E. Kiritsis, L. Mazzanti, G. Michalogiorgakis and F. Nitti, Improved Holographic QCD, Lect. Notes Phys. 828 (2011) 79 [arXiv:1006.5461] [InSPIRE].

[24] M. Alfimov, N. Gromov and V. Kazakov, QCD Pomeron from AdS/CFT Quantum Spectral Curve, JHEP 07 (2015) 164 [arXiv: 1408.2530] [INSPIRE].

[25] O. Bergman, G. Lifschytz and M. Lippert, Holographic Nuclear Physics, JHEP 11 (2007) 056 [arXiv: 0708.0326] [INSPIRE].

[26] S. Baldino, S. Bolognesi, S.B. Gudnason and D. Koksal, Solitonic approach to holographic nuclear physics, Phys. Rev. D 96 (2017) 034008 [arXiv:1703.08695] [INSPIRE].

[27] S.A. Hartnoll, C.P. Herzog and G.T. Horowitz, Building a Holographic Superconductor, Phys. Rev. Lett. 101 (2008) 031601 [arXiv:0803.3295] [INSPIRE].

[28] S.A. Hartnoll, Lectures on holographic methods for condensed matter physics, Class. Quant. Grav. 26 (2009) 224002 [arXiv:0903.3246] [INSPIRE].

[29] C.P. Herzog, Lectures on Holographic Superfluidity and Superconductivity, J. Phys. A 42 (2009) 343001 [arXiv:0904.1975] [INSPIRE]. 
[30] C.P. Herzog, An Analytic Holographic Superconductor, Phys. Rev. D 81 (2010) 126009 [arXiv: 1003.3278] [INSPIRE].

[31] J. McGreevy, Holographic duality with a view toward many-body physics, Adv. High Energy Phys. 2010 (2010) 723105 [arXiv: 0909. 0518] [INSPIRE].

[32] T. Nishioka, S. Ryu and T. Takayanagi, Holographic Superconductor/Insulator Transition at Zero Temperature, JHEP 03 (2010) 131 [arXiv:0911.0962] [INSPIRE].

[33] M. Cubrovic, J. Zaanen and K. Schalm, String Theory, Quantum Phase Transitions and the Emergent Fermi-Liquid, Science 325 (2009) 439 [arXiv:0904.1993] [INSPIRE].

[34] H. Liu, J. McGreevy and D. Vegh, Non-Fermi liquids from holography, Phys. Rev. D 83 (2011) 065029 [arXiv:0903.2477] [INSPIRE].

[35] N. Iqbal, H. Liu and M. Mezei, Lectures on holographic non-Fermi liquids and quantum phase transitions, in Theoretical Advanced Study Institute in Elementary Particle Physics: String theory and its Applications: From meV to the Planck Scale, World Scientific (2011) [DOI] [arXiv:1110.3814] [INSPIRE].

[36] T. Faulkner and J. Polchinski, Semi-Holographic Fermi Liquids, JHEP 06 (2011) 012 [arXiv: 1001.5049] [INSPIRE].

[37] R.-G. Cai, L. Li, L.-F. Li and R.-Q. Yang, Introduction to Holographic Superconductor Models, Sci. China Phys. Mech. Astron. 58 (2015) 060401 [arXiv:1502.00437] [INSPIRE].

[38] A.A. Abrikosov, L.P. Gorkov and I.E. Dzyaloshinski, Methods of Quantum Field Theory in Statistical Physics, Courier Corporation (2012).

[39] E. Lifshitz and L.P. Pitaevskii, Statistical Physics: Theory of the Condensed State, vol. 9 of Course of Theoretical Physics, Butterworth-Heinemann (2013).

[40] E.H. Hall, On a new action of the magnet on electric currents, Am. J. MAth. 2 (1879) 287.

[41] K. von Klitzing, G. Dorda and M. Pepper, New method for high accuracy determination of the fine structure constant based on quantized Hall resistance, Phys. Rev. Lett. 45 (1980) 494 [INSPIRE].

[42] X.L. Qi and S.C. Zhang, Topological insulators and superconductors, Rev. Mod. Phys. 83 (2011) 1057 [arXiv: 1008. 2026] [inSPIRE].

[43] M.Z. Hasan and C.L. Kane, Topological Insulators, Rev. Mod. Phys. 82 (2010) 3045 [arXiv: 1002.3895] [INSPIRE].

[44] G.H. Wannier, Theorem on the magnetoconductivity of metals, Phys. Rev. B 5 (1972) 3836.

[45] H.-J. Kim et al., Dirac versus Weyl Fermions in Topological Insulators: Adler-Bell-Jackiw Anomaly in Transport Phenomena, Phys. Rev. Lett. 111 (2013) 246603 [arXiv:1307.6990] [INSPIRE].

[46] J. Xiong et al., Evidence for the chiral anomaly in the Dirac semimetal $N a_{3} B i$, Science $\mathbf{3 5 0}$ (2015) 413.

[47] H. Li et al., Negative magnetoresistance in Dirac semimetal $C d_{3} A s_{2}$, Nat. Commun. 7 (2016) 1 [arXiv:1507.06470].

[48] C. Zhang et al., Signatures of the Adler-Bell-Jackiw chiral anomaly in a Weyl Fermion semimetal, Nature Commun. 7 (2016) 0735 [arXiv: 1601.04208] [INSPIRE]. 
[49] B. Zhao et al., Weak antilocalization in $C d_{3} A s_{2}$ thin films, Sci. Rep. 6 (2016) 22377 [arXiv: 1601.05536].

[50] D.T. Son and B.Z. Spivak, Chiral Anomaly and Classical Negative Magnetoresistance of Weyl Metals, Phys. Rev. B 88 (2013) 104412 [arXiv:1206.1627] [InSPIRE].

[51] P. Hosur and X. Qi, Recent developments in transport phenomena in Weyl semimetals, Comptes Rendus Physique 14 (2013) 857 [arXiv:1309.4464] [INSPIRE].

[52] A.A. Burkov, Chiral anomaly and diffusive magnetotransport in Weyl metals, Phys. Rev. Lett. 113 (2014) 247203 [arXiv:1409.0013].

[53] A.A. Burkov, Negative longitudinal magnetoresistance in Dirac and Weyl metals, Phys. Rev. B 91 (2015) 245157 [arXiv: 1505.01849].

[54] H.-Z. Lu and S.-Q. Shen, Quantum transport in topological semimetals under magnetic fields, Front. Phys. 12 (2017) 127201 [arXiv:1609.01029].

[55] A. Jimenez-Alba, K. Landsteiner and L. Melgar, Anomalous magnetoresponse and the Stückelberg axion in holography, Phys. Rev. D 90 (2014) 126004 [arXiv:1407.8162] [INSPIRE].

[56] A. Jimenez-Alba, K. Landsteiner, Y. Liu and Y.-W. Sun, Anomalous magnetoconductivity and relaxation times in holography, JHEP 07 (2015) 117 [arXiv:1504.06566] [INSPIRE].

[57] K. Landsteiner, Y. Liu and Y.-W. Sun, Negative magnetoresistivity in chiral fluids and holography, JHEP 03 (2015) 127 [arXiv: 1410.6399] [INSPIRE].

[58] Y.-W. Sun and Q. Yang, Negative magnetoresistivity in holography, JHEP 09 (2016) 122 [arXiv: 1603.02624] [INSPIRE].

[59] P.A. Lee, N. Nagaosa and X.-G. Wen, Doping a Mott insulator: Physics of high-temperature superconductivity, Rev. Mod. Phys. 78 (2006) 17 [cond-mat/0410445] [INSPIRE].

[60] M. Edalati, R.G. Leigh and P.W. Phillips, Dynamically Generated Mott Gap from Holography, Phys. Rev. Lett. 106 (2011) 091602 [arXiv: 1010.3238] [INSPIRE].

[61] M. Edalati, R.G. Leigh, K.W. Lo and P.W. Phillips, Dynamical Gap and Cuprate-like Physics from Holography, Phys. Rev. D 83 (2011) 046012 [arXiv: 1012.3751] [InSPIRE].

[62] X.-M. Kuang, E. Papantonopoulos, B. Wang and J.-P. Wu, Dynamically generated gap from holography in the charged black brane with hyperscaling violation, JHEP 04 (2015) 137 [arXiv: 1411.5627] [INSPIRE].

[63] Y. Ling, P. Liu, C. Niu, J.-P. Wu and Z.-Y. Xian, Holographic fermionic system with dipole coupling on Q-lattice, JHEP 12 (2014) 149 [arXiv:1410.7323] [INSPIRE].

[64] Y. Ling, P. Liu, C. Niu and J.-P. Wu, Building a doped Mott system by holography, Phys. Rev. D 92 (2015) 086003 [arXiv: 1507.02514] [INSPIRE].

[65] Y. Ling, P. Liu and J.-P. Wu, A novel insulator by holographic Q-lattices, JHEP 02 (2016) 075 [arXiv: 1510.05456$]$ [INSPIRE].

[66] M. Fujita, S. Harrison, A. Karch, R. Meyer and N.M. Paquette, Towards a Holographic Bose-Hubbard Model, JHEP 04 (2015) 068 [arXiv: 1411.7899] [INSPIRE].

[67] E. Kiritsis and J. Ren, On Holographic Insulators and Supersolids, JHEP 09 (2015) 168 [arXiv: 1503.03481] [INSPIRE]. 
[68] M. Baggioli and O. Pujolàs, On Effective Holographic Mott Insulators, JHEP 12 (2016) 107 [arXiv: 1604.08915] [INSPIRE].

[69] S. Cremonini, A. Hoover and L. Li, Backreacted DBI Magnetotransport with Momentum Dissipation, JHEP 10 (2017) 133 [arXiv:1707.01505] [INSPIRE].

[70] D. Vegh, Holography without translational symmetry, arXiv:1301.0537 [INSPIRE].

[71] R.-G. Cai, Y.-P. Hu, Q.-Y. Pan and Y.-L. Zhang, Thermodynamics of Black Holes in Massive Gravity, Phys. Rev. D 91 (2015) 024032 [arXiv:1409.2369] [InSPIRE].

[72] A. Donos and J.P. Gauntlett, Novel metals and insulators from holography, JHEP 06 (2014) 007 [arXiv: 1401.5077] [INSPIRE].

[73] M. Blake and A. Donos, Quantum Critical Transport and the Hall Angle, Phys. Rev. Lett. 114 (2015) 021601 [arXiv: 1406.1659] [INSPIRE].

[74] S.A. Hartnoll and P. Kovtun, Hall conductivity from dyonic black holes, Phys. Rev. D 76 (2007) 066001 [arXiv:0704.1160] [INSPIRE].

[75] X. Guo, P. Wang and H. Yang, Membrane Paradigm and Holographic DC Conductivity for Nonlinear Electrodynamics, Phys. Rev. D 98 (2018) 026021 [arXiv:1711.03298] [InSPIRE].

[76] R.A. Davison and B. Goutéraux, Dissecting holographic conductivities, JHEP 09 (2015) 090 [arXiv: 1505.05092] [INSPIRE].

[77] P. Wang, H. Wu and H. Yang, Holographic DC Conductivity for Backreacted Nonlinear Electrodynamics with Momentum Dissipation, Eur. Phys. J. C 79 (2019) 6 [arXiv: 1805.07913] [INSPIRE].

[78] S.C. Zhang, T.H. Hansson and S. Kivelson, An effective field theory model for the fractional quantum hall effect, Phys. Rev. Lett. 62 (1988) 82 [INSPIRE].

[79] E. Fradkin and A. Lopez, Fractional Quantum Hall effect and Chern-Simons gauge theories, Phys. Rev. B 44 (1991) 5246 [INSPIRE].

[80] S.-C. Zhang, The Chern-Simons-Landau-Ginzburg theory of the fractional quantum Hall effect, Int. J. Mod. Phys. B 6 (1992) 25 [InSPIRE].

[81] D. Tong, Lectures on the Quantum Hall Effect, arXiv:1606.06687 [INSPIRE].

[82] M. Born, Modified field equations with a finite radius of the electron, Nature 132 (1933) 282.

[83] M. Born, Quantum theory of the electromagnetic field, Proc. Roy. Soc. Lond. A 143 (1934) 410 [INSPIRE].

[84] B. Zwiebach, A First Course in String Theory, Cambridge University Press (2009) [DOI]. 